\title{
Ex Vivo Adenoviral Vector-Mediated Neurotrophin Gene Transfer to Olfactory Ensheathing Glia: Effects on Rubrospinal Tract Regeneration, Lesion Size, and Functional Recovery after Implantation in the Injured Rat Spinal Cord
}

\author{
Marc J. Ruitenberg, ${ }^{1 \star}$ Giles W. Plant, ${ }^{2,3 *}$ Frank P. T. Hamers, ${ }^{4}$ Joke Wortel, ${ }^{1}$ Bas Blits, ${ }^{1}$ Paul A. Dijkhuizen, ${ }^{1}$ \\ Willem Hendrik Gispen, ${ }^{4}$ Gerard J. Boer, ${ }^{1}$ and Joost Verhaagen ${ }^{1}$ \\ ${ }^{1}$ Graduate School for Neurosciences Amsterdam, Netherlands Institute for Brain Research, 1105 AZ, Amsterdam, The Netherlands, ${ }^{2}$ Red's Spinal Cord \\ Research Laboratory, School of Anatomy and Human Biology, Clinical Training and Education Center Building, and ${ }^{3}$ Western Australian Institute for \\ Medical Research, The University of Western Australia, Crawley, Perth, WA 6009, Australia, and ${ }^{4}$ Department of Anatomy and Pharmacology, Rudolf \\ Magnus Institute for Neurosciences, 3584 CG, Utrecht, The Netherlands
}

\begin{abstract}
The present study uniquely combines olfactory ensheathing glia (OEG) implantation with ex vivo adenoviral (AdV) vector-based neurotrophin gene therapy in an attempt to enhance regeneration after cervical spinal cord injury. Primary OEG were transduced with AdV vectors encoding rat brain-derived neurotrophic factor (BDNF), neurotrophin-3 (NT-3), or bacterial marker protein $\beta$-galactosidase ( LacZ) and subsequently implanted into adult Fischer rats directly after unilateral transection of the dorsolateral funiculus. Implanted animals received a total of $2 \times 10^{5} \mathrm{OEG}$ that were subjected to transduction with neurotrophin-encoding AdV vector, AdV-LacZ, or no vector, respectively. At 4 months after injury, lesion volumes were smaller in all OEG implanted rats and significantly reduced in size after implantation of neurotrophin-encoding AdV vector-transduced OEG. All OEG grafts were filled with neurofilament-positive axons, and AdV vector-mediated expression of BDNF by implanted cells significantly enhanced regenerative sprouting of the rubrospinal tract. Behavioral analysis revealed that OEG-implanted rats displayed better locomotion during horizontal rope walking than unimplanted lesioned controls. Recovery of hind limb function was also improved after implantation of OEG that were transduced with a BDNF- or NT-3-encoding AdV vector. Hind limb performance during horizontal rope locomotion did directly correlate with lesion size, suggesting that neuroprotective effects of OEG implants contributed to the level of functional recovery. Thus, our results demonstrate that genetic engineering of OEG not only resulted in a cell that was more effective in promoting axonal outgrowth but could also lead to enhanced recovery after injury, possibly by sparing of spinal tissue.
\end{abstract}

Key words: functional recovery; gene therapy; neuroprotection; olfactory ensheathing glia; regeneration; rubrospinal tract; spinal cord injury; viral vectors

\section{Introduction}

Impairment in voluntary motor function after spinal trauma has often been attributed to disruption of descending motor pathways at the injury site (Nathan, 1994). After such damage, very little regenerative response occurs, resulting in a permanent loss

\footnotetext{
Received Feb. 12, 2003; revised June 3, 2003; accepted June 6, 2003.

This work was supported by Netherlands Organization for Scientific Research (NW0-GMW) Pioneer Grant 03094-142 and New Drug Research Foundation Research Grant 014-80-010 (J.V.), and National Health and Medica Research Council Research Grant 9935975, Neurotrauma Research Program Research Grant GNT 005/006, and the Ramaciotti Foundation (G.W.P.). We are grateful to Dr.P. Wood for providing the $775^{\text {NTR }}$ monoclonal antibody, and C. Christensen and R. Eggers for excellent technical support. The $2 \mathrm{H} 3$ monoclonal antibody, developed by Drs. T. M. Jessel and J. Dodd, was obtained from the Developmental Studies Hybridoma Bank, developed under the auspices of the National Institute of Child Health and Human Development, and maintained by The University of lowa Department of Biological Sciences. We acknowledge Dr. R. J. Pasterkamp for critical reading and valuable comments on this manuscript, and $\mathrm{G}$. van der Meulen for technical assistance with preparation of the artwork.

*M.J.R. and G.W.P. contributed equally to this work.

Correspondence should be addressed to J. Verhaagen, Netherlands Institute for Brain Research, Meibergdreef 33, 1105 AZ, Amsterdam Z0, The Netherlands. E-mail: j.verhaagen@nih.knaw.nl.

Copyright $\odot 2003$ Society for Neuroscience $\quad$ 0270-6474/03/237045-14\$15.00/0
}

of function and paralysis. This paucity of regeneration in the adult mammalian CNS is thought to depend on a negative balance between factors that inhibit or promote axon growth (for review, see Schwab and Bartholdi, 1996; Fournier and Strittmatter, 2001). Neurotrophic factor delivery has been studied extensively to overcome inhibition and augment regeneration of spinal motor pathways, such as the corticospinal tract (CST) and rubrospinal tract (RST) (for review, see Jones et al., 2001; Murray and Fischer, 2001).

Neurotrophins are well known for their beneficial effects on neuroprotection and neurite outgrowth. Local delivery of neurotrophins can counteract pathological events and induce a regenerative response after both acute (Schnell et al., 1994; Tetzlaff et al., 1994) and chronic (Houle and Ye, 1999; Kwon et al., 2002) spinal cord injury. In addition to intraparenchymal infusions, genetically modified cells that deliver neurotrophins have been studied. Engineered fibroblasts (Grill et al., 1997; Liu et al., 1999), Schwann cells (Menei et al., 1998), and pieces of peripheral nerve 
(Blits et al., 2000) have been used to induce regeneration after injury. Recently, much research has focused on the use of olfactory ensheathing glia (OEG) that may have advantages over other cellular conduits (for review, see Franklin and Barnett, 2000; Plant et al., 2000).

In the adult olfactory neuroepithelium, dying receptor neurons are replaced from a compartment of stem cells in the basal region of the epithelium (Farbman, 1992). The ability of new primary olfactory neurons to elongate their axons into a CNS environment is thought to be related to the presence of OEG (Doucette, 1990, 1991). These cells uniquely present both Schwann cell-like and astrocyte-like characteristics (for review, see Ramon-Cueto and Avila, 1998) and seem to have great potential to repair the damaged spinal cord (Li et al., 1997, 1998; Ramon-Cueto et al., 1998; Barnett et al., 2000; Ramon-Cueto et al., 2000; Lu et al., 2001a). However, not all axonal populations grow into OEG implants (Gudino-Cabrera et al., 2000). Additional adenoviral (AdV) vector-mediated neurotrophin expression could enhance their regeneration-supporting properties, because endogenous neurotrophic factor expression by OEG is low (Boruch et al., 2001; Woodhall et al., 2001). AdV vector-mediated gene transfer to OEG results in transient transgene expression for $\sim 30 \mathrm{~d}$ after implantation in the lesioned rat spinal cord (Ruitenberg et al., 2002).

The present experiment represents a new approach to spinal cord repair by applying ex vivo AdV vector-transduced OEG implants in an attempt to create more conducive conditions for RST regeneration. We examined the effects of AdV vector-mediated hypersecretion of brain-derived neurotrophic factor (BDNF) and neurotrophin-3 (NT-3), two putative RST neurotrophic factors (Kobayashi et al., 1997; Liebl et al., 2001), on functional recovery and RST regeneration after cervical injury. Anatomical, electrophysiological, and functional analysis was used to assess regeneration in animals over a 4 month observation period.

\section{Materials and Methods}

Recombinant $A d V$ stock production. The production of first generation $E 1$-deleted adenoviral vector stocks encoding the bacterial marker enzyme $\beta$-galactosidase ( $\beta$-gal; AdV-LacZ) (Hermens et al., 1997) or rat NT-3 (AdV-NT-3) (Dijkhuizen et al., 1997) has been described previously in detail. For the construction of an AdV vector-encoding BDNF, total RNA was isolated from adult rat brain. First strand cDNA was synthesized using reverse transcriptase (Invitrogen, Grand Island, NY) and oligo-dT primers. Subsequently, BDNF cDNA was amplified from the total cDNA via a PCR using an upstream sense primer $5^{\prime}$-CCC GGA ATT CGC CAC CAT GAC CAT CCT TTT CCT TAC T- $3^{\prime}$ and downstream antisense primer $5^{\prime}$-TTT AGA ATT CCT ATC TTC CCC TTT TAA TTG GT-3' (Amersham Biosciences, Piscataway, NJ). Primer sets were designed such that an EcoR1 site and a Kozak sequence (GCCACC; Kozak, 1987) were introduced in front of the translation startcodon ATG. The PCR product was then subcloned in the expression plasmid pcDNA-I/Amp (Invitrogen) downstream of the human cytomegalovirus (CMV) promoter. Nucleic acid sequencing was performed to identify the PCR product as rat BDNF cDNA using the United States Biochemicals sequencing kit (United States Biochemicals, Cleveland, OH). Next, BDNF cDNA was cloned in the AdV-targeting plasmid pAd309 dlE1.sl/ SalI + , yielding the plasmid pAd-BDNF. All targeting plasmids, encoding LacZ, BDNF, or NT-3, respectively, contained a transgene expression cassette with the CMV promoter to drive transgene expression and an SV-40 splice donor-acceptor site and polyadenylation sequence downstream of the gene of interest.

Replication-deficient E1-deleted AdV vectors were produced using standard procedures (Akli et al., 1993). In brief, AdV-targeting plasmid was linearized by SalI digestion. Linearized targeting plasmid was then cotransfected with ClaI- and XbaI-digested Ad5 DNA into 911 producer cells (Fallaux et al., 1996). The following day, cells were overlain with
$0.66 \%$ agar-containing medium (Invitrogen). After $8 \mathrm{~d}$, lytic plaques were isolated and characterized by PCR. Plaque-purified recombinant AdV vector was then expanded on 911 cells, harvested, and purified by two rounds of $\mathrm{CsCl}$ density gradient centrifugation. Recombinant AdV vector particles were collected from the gradient, dialyzed against TS buffer $\left(25 \mathrm{~mm}\right.$ Tris-HCl, $0.7 \mathrm{~mm} \mathrm{Na} \mathrm{HPO}_{4}, 137 \mathrm{~mm} \mathrm{NaCl}, 6 \mathrm{~mm} \mathrm{KCl}, 1.1$ $\mathrm{mm} \mathrm{MgCl} 2,0.9 \mathrm{mM} \mathrm{CaCl}_{2}$ ), $\mathrm{pH} 7.4$, and stored in small aliquots at $-80^{\circ} \mathrm{C}$ in TS buffer containing $10 \%$ glycerol. Viral stock titers were determined by a plaque assay on 911 cells and expressed as plaque-forming units per milliliter ( $\mathrm{pfu} / \mathrm{ml}$ ). All stock titers used were in the range of $10^{10} \mathrm{pfu} / \mathrm{ml}$. Before use, recombinant AdV vector stocks were screened for the absence of replication-competent adenovirus as described previously (Hermens et al., 1997).

Culturing of OEG. The isolation of OEG from the olfactory bulb nerve layer has been described previously (Yan et al., 2001). Briefly, adult female Fischer F344 rats were killed by intraperitoneal injection of a lethal dose of Nembutal ( Rhône-Mérieux, Pinkenba, QLD, Australia) and decapitated. The olfactory bulbs were dissected and rapidly transferred to Leibovitz's-15 (L-15) medium (Sigma, St Louis, MO). The pia was then carefully removed under a microscope using fine Dumont forceps. Next, the ventral olfactory nerve and glomerular layers were dissected away from the rest of the bulb and cut into $1 \mathrm{~mm}^{3}$ pieces. Subsequently, tissue blocks were incubated with $0.25 \%$ trypsin (Invitrogen) containing 50 $\mathrm{mg} / \mathrm{ml} \mathrm{DNase} \mathrm{(Invitrogen)} \mathrm{at} 37^{\circ} \mathrm{C}$ for $60 \mathrm{~min}$ with continual shaking. Trypsinization was stopped by adding DMEM (Sigma) and Ham's F-12 (DMEM/F-12; 1:1 mixture; Sigma) supplemented with 10\% fetal bovine serum (FBS; df-10S; Invitrogen) and $50 \mathrm{mg} / \mathrm{ml}$ gentamicin (Invitrogen). The suspension was then gently triturated and plated onto poly-L-lysinecoated $10 \mathrm{~cm}$ dishes. Six to seven days after the initial plating, OEG were purified from contaminated cells by immunoaffinity using the p75 neurotrophin receptor $\left(\mathrm{p} 75^{\mathrm{NTR}}\right)$. For this, $10 \mathrm{~cm}$ Petri dishes (Scot Scientific, Perth, WA, Australia) were incubated overnight at $4^{\circ} \mathrm{C}$ with goat antimouse antibodies (1:100; ICN Biomedicals, Seven Hills, NSW, Australia). After several washes, the dishes were incubated with $\mathrm{p} 75^{\mathrm{NTR}}$ monoclonal antibody (generously provided by Dr. P. Wood, University of Miami School of Medicine, Miami, FL) in L-15 medium supplemented with $5 \%$ FBS for $2 \mathrm{hr}$ at $4^{\circ} \mathrm{C}$. Unbound antibody was removed by several washings with L-15 medium. The cells in primary culture were then detached by trypsinization, pelleted by centrifugation, and resuspended in L-15 medium. Next, a $10 \mathrm{ml}$ suspension of olfactory bulb cells was plated onto pretreated dishes at a density of $4 \times 10^{5}$ cells per dish for 30 min at $4^{\circ} \mathrm{C}$. Unbound cells were removed from the dishes during several washes with L-15 medium. The attached cells were carefully dislodged from the dishes using a cell scraper (Sarstedt, Newton, NC), centrifuged, and resuspended in $\mathrm{df}-10 \mathrm{~S}$. Purified cells were then seeded onto poly-Llysine-coated $10 \mathrm{~cm}$ dishes and fed with $\mathrm{df}-10 \mathrm{~S}$ containing mitogens $(20$ $\mu \mathrm{g} / \mathrm{ml}$ pituitary extract and $2 \mu \mathrm{M}$ forskolin; Sigma).

Transduction of OEG cultures with AdV vectors. The methods for optimal transduction of primary OEG using AdV vector-mediated gene transfer have been described recently (Ruitenberg et al., 2002). Briefly, cells were seeded onto poly-L-lysine-coated six-well plates at a density of $10^{5}$ cells per well. OEG were then fed overnight with df-10S supplemented with mitogens (see above). The following day, the medium was replaced with df-10S containing a lowered mitogen concentration (2 $\mu \mathrm{g} / \mathrm{ml}$ pituitary extract and $0.2 \mu \mathrm{M}$ forskolin) to minimize OEG proliferation. Because AdV vectors do not integrate into the host cell genome, proliferation of transduced OEG will lead to the loss of episomally located viral DNA and, consequently, transgene expression. Recombinant AdV vector encoding $\beta$-gal, BDNF, or NT-3 was added to each well with a multiplicity of infection (moi) of 100, resulting in the transduction of virtually all cultured cells (Ruitenberg et al., 2002). Three days after infection, OEG cultures were fixed with $4 \%$ paraformaldehyde (PFA) and $0.2 \%$ glutaraldehyde in PBS for $10 \mathrm{~min}$ at room temperature (RT). Transduced OEG cultures were then subjected to nonradioactive in situ hybridization analysis, as described by Giger et al. (1996), using digoxigenin (DIG)-labeled antisense cRNA probes specific for $\beta$-gal, BDNF, or NT-3.

Neurotrophin production from transduced OEG. Conditioned medium was collected from transduced OEG cultures to determine the levels of 
secreted AdV vector-derived BDNF and NT-3 via an ELISA, as described by Woodhall et al. (2001). Transduced cultures of OEG were prepared as described above. Three days after infection, the medium was refreshed and the cultures were left for $24 \mathrm{hr}$, allowing determination of net production levels per day. Samples of conditioned medium taken from control and transduced OEG cultures were rapidly frozen in dry ice-ethanol and stored at $-80^{\circ} \mathrm{C}$ until additional processing.

The Emax immunoassay system (Promega, Leiden, The Netherlands) was used to determine the levels of neurotrophic factor protein in OEGconditioned medium according to the instructions of the manufacturer. The amount of secreted neurotrophic factor was expressed as nanograms of BDNF or NT-3 derived from a transduced OEG culture $\left(10^{5}\right.$ cells $)$ per day.

Biological activity of AdV vector-derived neurotrophins. To determine whether recombinant BDNF and NT-3 protein was biologically active, we studied their effect on neurite outgrowth from embryonic dorsal root ganglion (DRG) explants as described by Dijkhuizen et al. (1997). Purified OEG $\left(10^{4}\right.$ cells $)$ were seeded onto poly-L-lysine-coated $12 \mathrm{~mm}$ glass coverslips and infected with AdV-LacZ, AdV-BDNF, AdV-NT-3, or no virus, respectively, as described above.

Three days after AdV vector-mediated transduction of OEG cultures, embryonic DRGs were removed from timed pregnant female rats. Briefly, embryonic day (E) 14 pregnant females were killed by an intraperitoneal overdose of Nembutal and decapitated. Next, the entire litter was rapidly removed by Cesarean section and transferred to wet icecooled L-15 medium. E14 lumbar DRGs were then aseptically dissected from rat embryos and pooled in ice-cold L-15 medium. Isolated DRGs were subsequently transferred to DMEM/F-12 medium (1:1 mixture), and the medium from OEG cultures was removed by aspiration. Single DRGs were taken up in $50 \mu \mathrm{l}$ of DMEM/F-12 medium and plated on top of AdV vector-transduced OEG monolayers. Cocultures of OEG and DRG explants were grown for an additional $24 \mathrm{hr}$ in a $\mathrm{CO}_{2}$ incubator $(5 \%)$ at $37^{\circ} \mathrm{C}$.

To visualize neurite outgrowth, cocultures were fixed with $4 \%$ PFA in PBS for $30 \mathrm{~min}$. Fixed cultures were subsequently washed several times with Tris-buffered saline (TBS; $10 \mathrm{~mm}$ Tris- $\mathrm{HCl}, 135 \mathrm{~mm} \mathrm{NaCl}$ ), $\mathrm{pH}$ 7.4, and permeabilized with TBS containing $0.3 \%$ Triton X-100 and 5\% FBS. Cultures were then incubated overnight with the mouse monoclonal antibody $2 \mathrm{H} 3$ against rat neurofilament (NF) (1:1000; Developmental Studies Hybridoma Bank, University of Iowa, Iowa City, IA) at $4^{\circ} \mathrm{C}$. The following day, cultures were washed three times with TBS and incubated with Cy3-conjugated secondary antibody [1:400; donkey anti-mouse (DAM)-Cy3; Jackson ImmunoResearch, West Grove, PA]. Finally, cocultures were mounted in Vectashield (Vector Laboratories, Burlingame, CA) and coverslipped. Digital photographic images of neurite outgrowth from each DRG explant were then captured using a Zeiss (Thornwood, NY) confocal laser-scanning microscope (CLSM).

Preparation of OEG for spinal implantation. Suspensions of OEG for implantation purposes were prepared as described previously (Ruitenberg et al., 2002). Primary p $75^{\text {NTR }}$-purified OEG were seeded onto polyL-lysine-coated $6 \mathrm{~cm}$ dishes at a density of $10^{6}$ cells per plate and grown overnight in df- $10 \mathrm{~S}$ containing $2 \mu \mathrm{g} / \mathrm{ml}$ of pituitary extract and $0.2 \mu \mathrm{M}$ forskolin. The following day, the medium was replaced with fresh medium containing either no AdV vector or $10^{8}$ pfu (moi, 100) AdV-LacZ, AdV-BDNF, AdV-NT-3, or AdV-BDNF plus AdV-NT-3, respectively, and the cells were left for $72 \mathrm{hr}$. OEG cultures were then prelabeled with $10 \mu \mathrm{g} / \mathrm{ml}$ of Hoechst 33342 dye (bis-benzimide; Sigma) and subsequently washed several times with L-15 medium. Next, the cells were detached by trypsinization and washed twice in serum-free DMEM/F-12 medium. Cells were pelleted by low-speed centrifugation, carefully resuspended, and diluted in the appropriate volume of DMEM/F-12 medium to obtain a suspension of $10^{5} \mathrm{OEG}$ per microliter, as determined by counting. The viability of OEG suspensions was determined by counting the percentage of dead cells using Trypan blue staining. In all cases, the viability of OEG suspensions before implantation was $>95 \%$. Suspensions were kept cool on wet ice during surgery.

Experimental design. A total of 68 adult female Fischer F344 rats (175200 gm; Harlan-Olac, Oxon, UK) were used in this experiment. All rats were housed under standard conditions, maintained in a $12 \mathrm{hr}$ light/dark cycle, and had ad libitum access to water and food. All experimental procedures were conducted in accordance with the guidelines of the local animal welfare committee for use and care of laboratory animals. Body weight of experimental animals was monitored during the experiment as a general measure of health. Animals were divided into six experimental groups. Experimental group $1(n=10)$, which serves as a control, was subjected to a unilateral transection of the dorsolateral funiculus of the cervical spinal cord. Rats in experimental groups 2, 3, 4, 5, and 6 received a similar spinal cord lesion but also an implant of either control or AdV vector-transduced OEG. Animals in groups 2 and 3 received an implant of uninfected OEG $(n=10)$ or AdV-LacZ-transduced OEG $(n=12)$, respectively. These groups served as additional controls. In groups 4,5 , and 6, OEG were subjected to infection with AdV vectors encoding BDNF or NT-3 before implantation. Rats in experimental groups 4 and 5 received an implant of AdV-BDNF $(n=12)$ or AdV-NT-3-transduced $(n=12)$ OEG, respectively. In group 6, OEG were subjected to infection with a 1:1 mixture of BDNF- and NT-3-encoding AdV vector and subsequently implanted in the lesioned spinal cord $(n=12)$. All rats were tested weekly for 4 months for recovery of hind limb performance. Rubrospinal projections in these animals were then anterogradely labeled using biotinylated dextran amine (BDA) as a tracer 2 weeks before the animals were killed. Rubrospinal motor-evoked potentials (MEPs) were recorded for all experimental animals before perfusion to study regeneration of RST axons and integrity of descending spinal pathways in general. Seven days after implantation, two animals were taken from groups 3-6 and evaluated for transgene expression.

Surgical procedures. Implantation of OEG into a unilateral cervical transection injury model involving the RST was described previously in detail (Ruitenberg et al., 2002). In brief, rats were deeply anesthetized by intramuscular injection of ketamine $(100 \mathrm{mg} / \mathrm{kg}$ of body weight; Nimatek, Eurovet, Bladel, The Netherlands) and xylazine $(10 \mathrm{mg} / \mathrm{kg}$ of body weight; Bayer, Leverkussen, Germany). Access to the spinal cord was obtained via dorsal laminectomy of the fourth cervical vertebra after splitting the neck musculature. To expose the spinal cord, a small incision was made through both dura and pia mater using a sharpened $30 \mathrm{ga}$ needle. After identification of the dorsal root entry zone and midline of the spinal cord, the left dorsolateral funiculus of the spinal cord was lesioned with a pair of microscissors as deep as $1 \mathrm{~mm}$ ventral to the spinal surface, taking care not to damage the dorsal roots. Such a lesion resulted in complete transection of the RST projection area and slightly damaged the spinal gray matter. Next, OEG suspensions were carefully injected at $1 \mathrm{~mm}$ distance, both proximal and distal, from the lesion cavity using a Harvard microinjection apparatus. Each injection delivered $10^{5}$ cells. After removal of the injector, muscles and skin were closed in separate layers. All animals received a postoperative subcutaneous injection of 2 $\mathrm{ml}$ physiological salt solution to compensate for blood loss and Finadyne (flunixinum, $2.4 \mathrm{mg} / \mathrm{kg}$ of body weight for $3 \mathrm{~d}$; Schering-Plough, Brussels, Belgium) to suppress pain. At the end of surgical procedures, the viability of OEG suspensions used for implantation still ranged between 90 and $95 \%$, as determined by Trypan blue staining.

Locomotor analysis. After surgery, spinal cord-injured rats were allowed to recover for a period of $7 \mathrm{~d}$ before the assessment of locomotor capacity. Functional performance of the impaired hind limb was investigated via the Basso-Beattie-Bresnahan (BBB) locomotor rating scale (Basso et al., 1995) and during horizontal rope walking (Kim et al., 2001). In both cases, gait analysis of spinal cord-injured rats was postoperatively determined by two independent investigators that were blinded to the treatment of the animal. The first functional assay, the BBB rating score, was composed of 21 operational definitions studying several aspects involved in the locomotion of quadrupedal animals. As a more sensitive test to assess behavioral deficits in partial spinal cord hemisection models, horizontal rope walking was used to study more specific aspects such as posture and balance during gait. In this test, animals were trained to walk across a 1.25-meter-long rope (diameter, $4 \mathrm{~cm}$ ) from one platform to another while the gait of the animal was studied. To successfully complete this task, hind limb weight support, precise paw placement, and adaptable balance were required. Animals were pretrained for a period of 5 weeks before the start of the experiment, such that a constant rope locomotion performance was obtained. Trained animals crossed the 
rope quickly with an occasional minor slip and no falls. Rats were scored for their general performance during rope locomotion using a deficit rating scale defined as follows: (0) normal rope locomotion with rare minor deficits; (1) close to normal locomotion with occasional lesion deficits; (2) able to cross the rope but with obvious lesion deficits such as inability to maintain consistent weight-supported limb placement during traverse; or (3) great difficulty to cross the rope, frequent slips, and falls. To determine ratings, rats were given two conditioning trials and subsequently evaluated for forelimb and hindlimb placement together with general balance and posture during traverse. As they moved along the rope, all rats were also videotaped from a stationary lateral view, allowing us to determine the "total error/step" ratio. This quantitative analysis method included counting the number of slips and falls during rope traverse. From these data, a total error/step ratio was calculated and defined as the following: [number of slips +2 (number of falls)]/total number of steps. Each rat was observed three times, and final scores were either the mean of three independent observations (quantitative analysis) or the cumulative deficit score of three runs (maximum deficit score of 9), according to Kim et al. (2001).

RST axon tracing. Anterograde labeling of the RST was performed as described previously (Antal et al., 1992), using biotin-dextran conjugates (10\% BDA, 10,000 molecular weight in PBS) (Molecular Probes, Leiden, The Netherlands) as a neuronal tracer, $14 \mathrm{~d}$ before perfusion. In brief, a small hole was drilled in the skull under deep ketamine-xylazine anesthesia while the animal was held in a stereotaxic frame. Glass pipettes with a tip diameter of $60 \mu \mathrm{m}$ were filled with BDA tracer that was subsequently delivered to the red nucleus using iontophoresis. The stereotaxic coordinates for tracer delivery were, with horizontal skull position, $6 \mathrm{~mm}$ posterior and $0.7 \mathrm{~mm}$ lateral to bregma and $6.8 \mathrm{~mm}$ deep from the dorsal surface of the brain. A positive direct current of $6 \mu \mathrm{A}\left(V_{\max }, 30 \mathrm{~V}\right)$ was used with a pulse duration of $5 \mathrm{sec}$ followed by $5 \mathrm{sec}$ intervals for a period of $20 \mathrm{~min}$. The current was then switched off and the injector was left in place for $5 \mathrm{~min}$ to allow diffusion of BDA from the injection spot. Polarity of the current was then changed, followed by the careful withdrawal of the glass capillary and suturing of the skin.

Electrophysiology. At the end of the experiment, after conclusion of the behavioral analysis at 4 months after injury, rubrospinal MEPs were recorded as described previously (Lankhorst et al., 1999) to evaluate possible RST axon regeneration. Briefly, animals were deeply anesthetized with hypnorm $(0.4 \mathrm{ml} / \mathrm{kg}$ of body weight, i.m.; Janssen Pharmaceutical LTD, Grove, Oxford, UK) containing $0.315 \mathrm{mg} / \mathrm{ml}$ of fentanyl citrate and $10 \mathrm{mg} / \mathrm{ml}$ of fluanisone. Hypnorm injections were repeated every $30 \mathrm{~min}$ after induction of the anesthesia. Subsequently, the animals were intubated and mechanically ventilated after a neuromuscular blockade with pancronium bromide $(1.25 \mathrm{mg} / \mathrm{kg}$ of body weight, i.v.; Organon, Oss, The Netherlands). Rubrospinal MEPs were elicited by direct bipolar stimulation (SNEX-100x; $20 \mathrm{~mm}$; Bilaney Consultants, Dusseldorf, Germany) of the lesioned right red nucleus (stereotaxic coordinates as for tracer delivery) and recorded below vertebra C5 via an epidurally located bipolar recording electrode consisting of thin gold plates and with an interpolar distance of $5 \mathrm{~mm}$. Threshold values were subsequently determined by slowly increasing the applied voltage. All MEPs used for analysis were measured at twice the threshold value. To ensure that evoked potentials were derived from the lesioned red nucleus and not, in part, the result of cross-stimulation of the intact RST, the right lateral funiculus was transected at the level of vertebrae C3. Such a lesion completely destroyed the RST that originated from the left red nucleus, yielding the MEP signal that was attributable to stimulation of the right red nucleus. Immediately after recording of rubrospinal MEPs, animals were killed by transcardial perfusion with $100 \mathrm{ml}$ of saline followed by $300 \mathrm{ml}$ of ice-cold 4\% PFA in PBS. Brains and spinal cords were carefully dissected, postfixed overnight in 4\% PFA solution, and stored in $0.1 \%$ PFA in PBS containing $0.01 \% \mathrm{NaN}_{3}$ at $4^{\circ} \mathrm{C}$ until additional use.

For MEP analysis, a total of 1024 traces were averaged to improve the signal/noise ratio. Within the mean trace, deviations from the signal baseline were considered as a peak if the top-valley voltage difference was at least three times as large as the SD of the noise. The amplitude and latency of the first (monosynaptic) positive peak, as well as the summed amplitudes of all peaks during the first $5 \mathrm{msec}$ (stimulus artifact was discarded), were determined and included in the analysis.

Histological analysis. To evaluate transgene expression $7 \mathrm{~d}$ after implantation, horizontal sections through the cervical spinal cord were cut on a cryostat and subjected to standard in situ hybridization using DIGlabeled cRNA antisense probes against BDNF or NT-3 mRNA. Consecutive sections were double labeled with $\mathrm{p} 75^{\mathrm{NTR}}$ to identify the nature of transgene-expressing cells and astrocyte marker glial fibrillary acidic protein (GFAP; 1:5000; Dako, Glostrup, Denmark). After perfusion of the experimental animals, at 4 months after injury, fixed spinal cords (cervical region, C2-C6) were washed several times with PBS, embedded in $10 \%$ gelatin (Difco, Detroit, MI), and postfixed for $48 \mathrm{hr}$ in $4 \% \mathrm{PFA}$ solution at $4^{\circ} \mathrm{C}$ (Griffioen et al., 1992). Four series of consecutive horizontal vibratome sections $(40 \mu \mathrm{m})$ were cut in PBS and collected in serial vials.

BDA-traced RST axons were visualized for quantification purposes by staining two alternate series of the horizontal spinal cord sections $(50 \%$ of all sections). In brief, sections were washed three times in TBS, followed by irreversible blocking of endogenous peroxidase activity with $10 \%$ methanol and $3 \% \mathrm{H}_{2} \mathrm{O}_{2}$ in TBS for $1 \mathrm{hr}$ at RT. Subsequently, sections were washed three times and incubated for 30 min with TBS supplemented with $5 \%$ FBS and $0.3 \%$ Triton X-100. Sections were then incubated overnight with avidin-biotin-peroxidase complex at $4^{\circ} \mathrm{C}(1$ : 800 avidin plus 1:800 biotin; PK-6100 Elite Vectastain ABC-kit; Vector Laboratories, Burlingame, CA). The following day, sections were washed three times in TBS and incubated with biotinylated tyramid (1:1000; Dako) for $10 \mathrm{~min}$ in TBS containing $0.01 \% \mathrm{H}_{2} \mathrm{O}_{2}$ to allow signal amplification. Sections were washed again three times and incubated with $A B C$ reagent for $1 \mathrm{hr}$ at RT. After several washes, sections were stained with $3,3^{\prime}$-diaminobenzidine tetrahydrochloride (DAB) in $50 \mathrm{~mm}$ Tris- $\mathrm{HCl}$, $\mathrm{pH} 7.4$, containing $0.01 \% \mathrm{H}_{2} \mathrm{O}_{2}$ and $0.2 \mathrm{mg} / \mathrm{ml}$ of $\left(\mathrm{NH}_{4}\right)_{2} \cdot \mathrm{SO}_{4} \cdot \mathrm{NiSO}_{4}$ resulting in a dark purple precipitate. Sections were subsequently dehydrated in graded series of ethanol, cleared in xylene, and finally embedded in Entellan (Merck, Amsterdam, The Netherlands). To better visualize the spatial relationship of BDA-traced RST axons with the lesion and implantation site, camera lucida drawings of representative sections through the injured dorsolateral funiculus were made.

The third set of sections was used to examine in more detail the spatial relationship of BDA-traced RST axons and the glial scar using DAB double staining as described previously (Buijs et al., 1999). To this end, free-floating sections were pretreated as described above and incubated overnight with $\mathrm{ABC}$ reagent to stain traced RST axons. Next, the DAB color reaction was stopped via three washes with TBS followed by a 10 min incubation with $10 \%$ methanol and $3 \% \mathrm{H}_{2} \mathrm{O}_{2}$ containing TBS to block peroxidase activity. Sections were then incubated overnight with a rabbit polyclonal antibody against GFAP (1:5000; Dako) at $4^{\circ} \mathrm{C}$. The following day, unbound antibodies were washed away with multiple washings in TBS, and sections incubated with a horseradish peroxidaseconjugated secondary antibody were directed against rabbit immunoglobulins (1:200; Dako). Sections were stained as described above using $\mathrm{DAB}$ as a chromogen but without the addition of $\left(\mathrm{NH}_{4}\right)_{2} \cdot \mathrm{SO}_{4} \cdot \mathrm{NISO}_{4}$. This results in the formation of a brown immunoprecipitate that can be clearly distinguished from dark purple-stained RST axons. Sections were mounted on gelatin-coated slides and dried overnight at RT. The sections were then briefly counterstained with $0.2 \%$ cresyl violet solution, dehydrated in ethanol, and finally embedded in Entellan as described above.

The fourth and final set of vibratome sections was used to either gain an impression of the severity of scar formation at the lesion site at 4 months after injury, or to visualize regenerating axons in the lesion area. For this, p $75^{\text {NTR }}$ immunoreactivity was used as a general marker to visualize implanted OEG at the lesion site and combined with staining for NF or fibronectin. The latter antibody will stain p $75^{\text {NTR }}$-negative meningeal cells and fibroblasts that may have invaded the lesion site (Pasterkamp et al., 1999). In brief, sections were washed three times with TBS and permeabilized with TBS containing $0.3 \%$ Triton X-100 and 5\% FBS to block nonspecific binding of antibodies. Sections were then incubated overnight with primary antibodies against $\mathrm{p} 75^{\mathrm{NTR}}$ (1:50; mouse monoclonal antibody clone 192; Chemicon, Harrow, UK) and fibronectin (1: 50; rabbit polyclonal antibody; Chemicon) or NF (1:100; NF-200; Sigma) 
at $4^{\circ} \mathrm{C}$. The following day, sections were washed with TBS and incubated with a mixture of fluorophore-conjugated secondary antibodies for $1 \mathrm{hr}$ at RT: swine anti-rabbit-FITC and DAM-Cy3 (1:400; Jackson ImmunoResearch). Sections were mounted on slides and immersed in Vectashield. Digital photographic images were captured using a Zeiss CLSM device.

Quantification of RST axon numbers and lesion size. To quantify regeneration or sprouting of traced RST axons, BDA-positive axonal profiles in the lesion area were counted as described by Blits et al. (2000). First, the proximal border of the scar was determined using a dark-field filter and considered as "point 0." Lesion borders were defined as a clear discontinuity of normal spinal cord cytoarchitecture, the absence of healthylooking spinal neurons, and a high density of small cells [i.e., implanted OEG and endogenous cells (e.g., inflammatory cells) that are known to invade spinal cord injury sites] as described previously (Takami et al., 2002; Plant et al., 2003). Fiber counts were made at fixed distances up to several millimeters proximal and distal to this point. For this, a $65 \mu \mathrm{m}$ width frame was projected on the horizontal sections at 3, 2, 1.5, 1, 0.75, $0.5,0.25$, and $0 \mathrm{~mm}$ proximal or distal to the proximal border of the lesion (point 0 ). All BDA-positive axons visible within this frame were then counted at $200 \times$ magnification by an independent investigator who was blinded to the treatment of the animals. To correct for variability in the tracing results, relative RST axon numbers were calculated by expressing the fiber counts at each distance as a percentage of traced RST axons at $3 \mathrm{~mm}$ proximal to point 0 .

To assess possible counteracting effects of different OEG implants on the ongoing secondary degeneration at the injury site, lesion volumes of all experimental animals were determined using a computerized image analysis station. In brief, the same sections used for RST axon quantification (described above; $50 \%$ of total sections) were studied under darkfield microscopy, and the border of the damaged area was determined using the same criteria as described above. Next, lesion areas were outlined while the observer was blinded to the experimental treatment. The outlined area contained both scar-associated cells as well as grafted OEG, and in a rare occasion, some small cysts if present (primarily in unimplanted lesioned control animals). The outlined areas of each animal were subsequently imported into Microsoft (Seattle, WA) Excel, summed, multiplied by the section thickness, and corrected for the total number of sections.

Statistical analysis. Data obtained from histological analysis and electrophysiology, as well as the calculated total error/step ratios, were analyzed for statistical differences between animal groups $(p<0.05)$ using two-way ANOVA and post hoc Newman-Keuls tests. Functional data from the rope locomotion-rating scale (deficit scores) were evaluated using nonparametric Kruskall-Wallis ANOVA test. The Pearson correlation test was used to investigate whether there were possible relationships between different outcome parameters and were considered statistically significant if $p<0.01$. All data incorporated in the analysis were obtained from animals that survived the entire study.

\section{Results}

\section{In vitro analysis of transgenic neurotrophin expression}

Transduction of primary p $75^{\mathrm{NTR}}$-purified OEG cultures with first-generation E1-deleted adenoviral vectors resulted in high levels of transgene expression in many cells as determined by in situ hybridization at $3 \mathrm{~d}$ after infection (data not shown). Numerous cells expressed high levels of $\beta$-gal, BDNF, or NT-3 mRNA, respectively, without signs of toxicity or cytopathological effects. No staining for any of the transgenes was observed in uninfected OEG that served as control cultures or after hybridization with sense probe.

Conditioned medium from transduced OEG cultures was analyzed for the presence of neurotrophins BDNF and NT-3 using an ELISA assay $4 \mathrm{~d}$ after transduction with AdV vectors (Fig. $1 \mathrm{~A}$ ). Low levels of BDNF were detected in medium samples $(n=4)$ taken from control $\left(0.45 \pm 0.17 \mathrm{ng} / 10^{5}\right.$ cells per day) or AdVLacZ-transduced OEG cultures $\left(0.57 \pm 0.13 \mathrm{ng} / 10^{5}\right.$ cells per
A
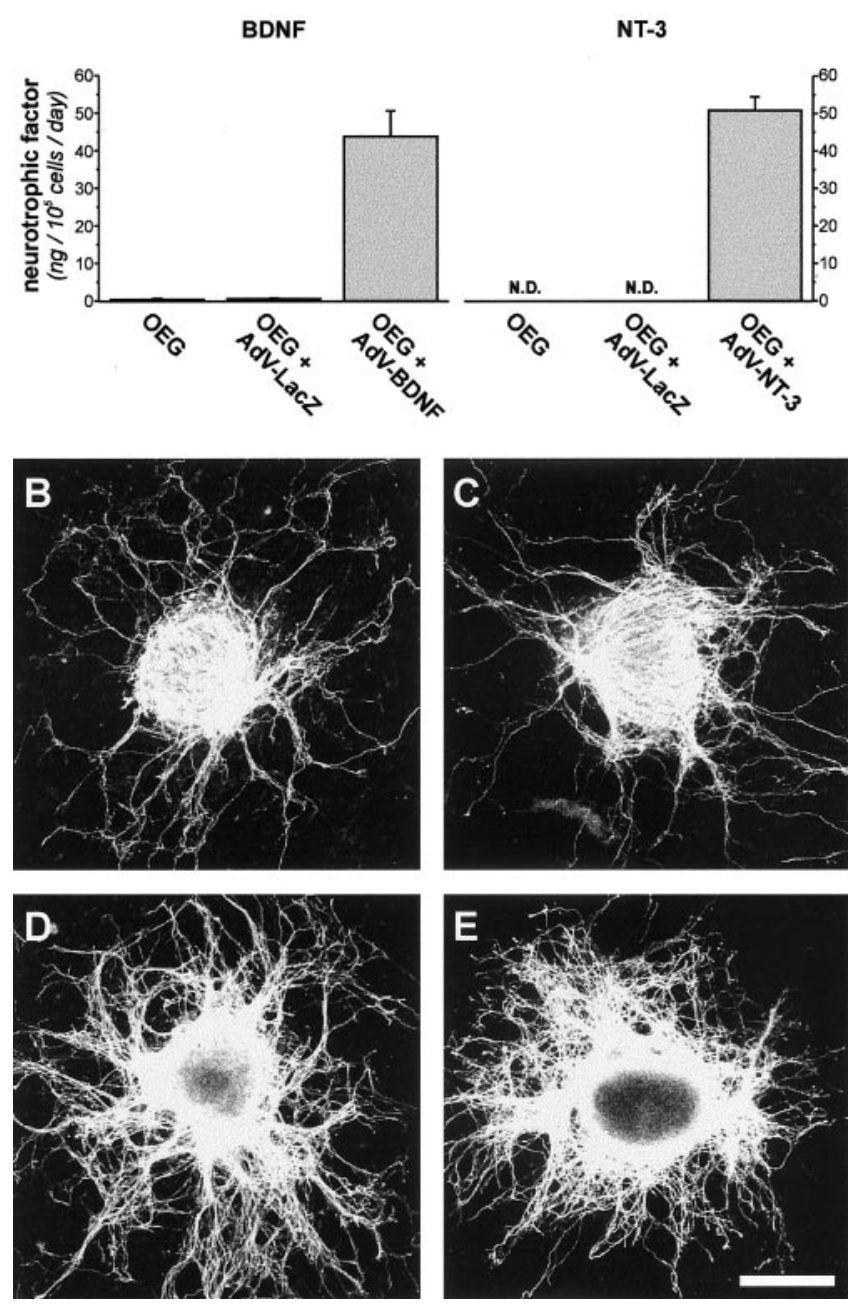

Figure 1. In vitro analysis of $\mathrm{AdV}$ vector-mediated transgene expression. $A$, Conditioned medium from uninfected and AdV-LacZ (controls) or neurotrophin-encoding AdV vectortransduced OEG cultures ( $10^{5}$ cells) was analyzed for the presence of BDNF and NT-3 using ELISA techniques. Bars indicate averages $\pm \operatorname{SEM}(n=4)$. At $4 \mathrm{~d}$ after transduction, low levels of BDNF $(\sim 0.5 \mathrm{ng}$ ) were found secreted in medium taken from control OEG cultures over a $24 \mathrm{hr}$ time period, whereas NT-3 was not detectable (N.D.). Transduction of OEG cultures with neurotrophin-encoding AdV vectors resulted in high levels of recombinant BDNF and NT-3, secreted into the culture medium at a net rate of $43.8 \pm 6.8 \mathrm{ng}$ and $50.8 \pm 3.7 \mathrm{ng} / 10^{5}$ cells per day, respectively. $B-E$, Biological activity of AdV vector-derived BDNF and NT-3 was confirmed in a $24 \mathrm{hr}$ coculture experiment of transduced OEG and E14 DRG explants. This allowed visualization of the additional effect of transgenic neurotrophin expression on the neurite outgrowthpromoting properties of OEG. Similar moderate outgrowth of neurites was observed when DRG explants were grown on top of uninfected $(B)$ or AdV-LacZ-transduced ( $C$ ) OEG cultures. This indicates that $A d V$ vector infection itself did not alter the growth-promoting properties of these cells. Robust neurite outgrowth was observed from DRG explants cocultured with OEG transduced with an AdV vector-encoding BDNF $(D)$ or NT-3 $(E)$. This demonstrates that AdV vector-derived neurotrophins were secreted from OEG in biologically active form, and that these transduced OEG were more effective in promoting neurite outgrowth in culture. Scale bar: (in $E) B-E, 1 \mathrm{~mm}$.

day), whereas NT-3 was not detectable. After infection with AdVBDNF, an $\sim 100$-fold increase in the levels of BDNF $(43.8 \pm 6.8$ ng) secreted from transduced OEG was observed. ELISA analysis of medium samples taken from AdV-NT-3-transduced OEG cultures revealed that at least $50.8 \pm 3.7 \mathrm{ng}$ of recombinant NT-3 was secreted from $10^{5}$ cells per day. These results indicate that, at the time-point of implantation, high amounts of recombinant neurotrophin were released from OEG transduced with an AdV vector-encoding BDNF or NT-3 as compared with controls. 
Biological activity of AdV vectorderived BDNF and NT-3 was demonstrated in a coculture experiment. For this, E14 DRG explants $(n=12)$ were positioned on top of an AdV vectortransduced monolayer of OEG to determine the effect of transgenic neurotrophic factor production and were subsequently analyzed for neurite outgrowth $24 \mathrm{hr}$ after the initial plating. Moderate extensions of neurites were observed when E14 DRG explants were cocultured with uninfected or AdV-LacZ-transduced OEG (Fig. $1 B, C$ ). No visible differences in radial neurite outgrowth were observed between these groups, indicating that AdV vector infection did not interfere with the growthsupporting properties of OEG. A robust outgrowth of neurites from DRG explants was found when OEG monolayers were transduced with an AdV vector-encoding BDNF or NT-3 (Fig. 1D,E). These results demonstrate that AdV vector-derived neurotrophins were biologically active and confirm that recombinant BDNF and NT-3 were secreted by transduced OEG as shown previously by ELISA.

In vivo analysis of transgene expression At $7 \mathrm{~d}$ after implantation, transgene expression in OEG implants was examined by in situ hybridization and immunohistochemical characterization of the lesion area (Fig. 2). Numerous cells displaying typical bipolar OEG morphology were detected in the lesion area expressing high levels of BDNF or NT-3 mRNA. No such staining was present in unimplanted animals or after implantation of AdV-LacZtransduced OEG (data not shown). To determine the identity of BDNF- and NT-3expressing cells, adjacent sections were examined for the presence of Hoechstlabeled cells and immunoreactivity for p $75^{\mathrm{NTR}}$, which is a marker for OEG. No BDNF- or NT-3-expressing cells were present outside the confines of $\mathrm{p} 75^{\mathrm{NTR}} \mathrm{im}$ munoreactivity and Hoechst distribution pattern, identifying them as being implanted OEG. The vast majority of BDNFand NT-3-positive cells were present in the inner core of the lesion, bridging the injury site as determined by GFAP staining that was used to visualize the developing glial scar. Some additional $\mathrm{p} 75^{\mathrm{NTR}}$ labeling was observed at the lateral edges of the spinal cord close to or in the dorsal root itself, indicating the presence of Schwann cells.

\section{Histological analysis}

Double staining for fibronectin and $\mathrm{p} 75^{\mathrm{NTR}}$ was used to visualize scar-associated cells, such as meningeal cells and fibroblasts, and implanted OEG, respectively. At 4 months after the injury, abundant fibronectin expression was found in unimplanted animals delineating the scar, but only a minor immunoreactivity for

p75

GFAP cytes. Scale bar, $100 \mu \mathrm{m}$.
BDNF
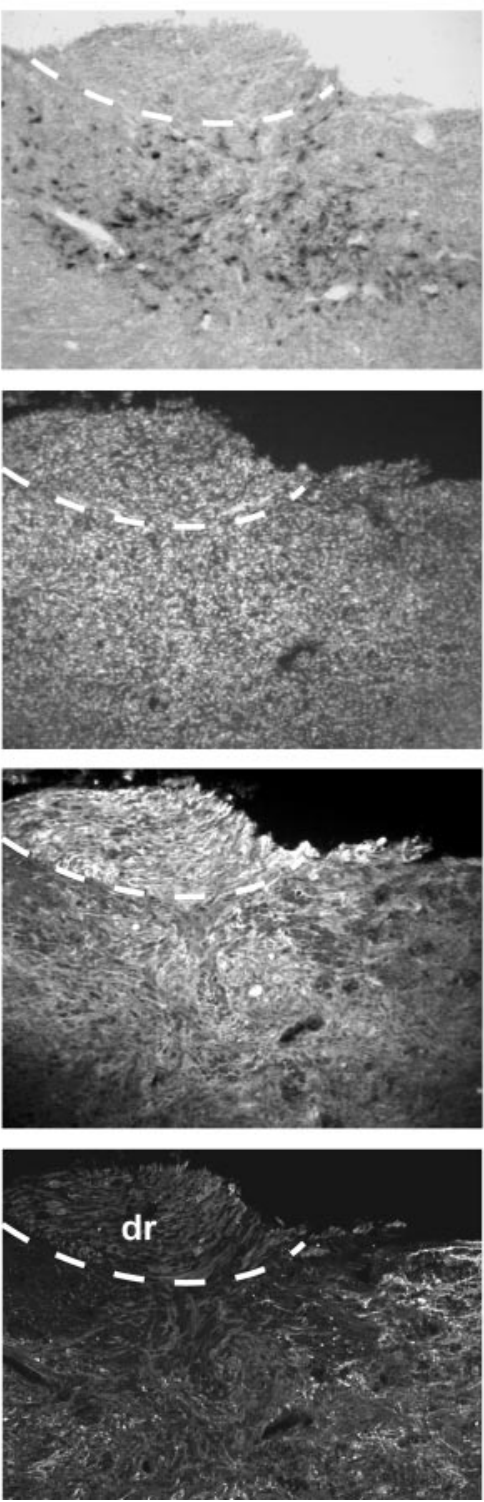

Figure 2. In vivo analysis of AdV vector-mediated neurotrophin expression by transduced OEG after implantation in the lesioned spinal cord. At $7 \mathrm{~d}$ after implantation, many cells expressing high levels of BDNF and NT-3 mRNA were detected in the lesion area. Animals implanted with uninfected or AdV-LacZ-transduced OEG (control implants) did not display such neurotrophin mRNA expression at the injury site. Transgene-expressing cells were nicely integrated in the host spinal cord bridging the injury site. Histological analysis of adjacent sections showed that these cells were detected within the confines of $p 75^{\text {NTR }}$ immunoreactivity, which identifies them as implanted OEG. Some additional labeling for p $75^{\text {NTR }}$ (Schwann cells) was found at the lateral edges of the spinal cord, at the level of the dorsal root (dr) entry zone (dashed line). Visualization of the developing glial scar showed that the vast majority of transgene-expressing cells was present in the lesion center and surrounded by GFAP-positive reactive astro-

p $75^{\text {NTR }}$ could be detected (Fig. $3 A$ ). The majority of these p75 ${ }^{\text {NTR }}$-labeled cells was found in the more lateral part of the lesion, close to the dorsal root entry zone. Most of these cells displayed typical bipolar morphology (Fig. $3 A$, inset), indicating that they were most likely Schwann cells that had migrated into the spinal cord after the injury. In contrast, high levels of $\mathrm{p} 75^{\text {NTR }}$ immunoreactivity were observed in all implanted animal groups, labeling many cells at the injury site but not rostral or caudal to it (Fig. 3B). This indicates that implanted OEG were able to survive in the spinal cord for at least 4 months after implantation, because only a few $\mathrm{p} 75^{\mathrm{NTR}}$-positive Schwann cells were found to invade the spinal cord. Clusters of aligned p $75^{\mathrm{NTR}}$-positive cells, 

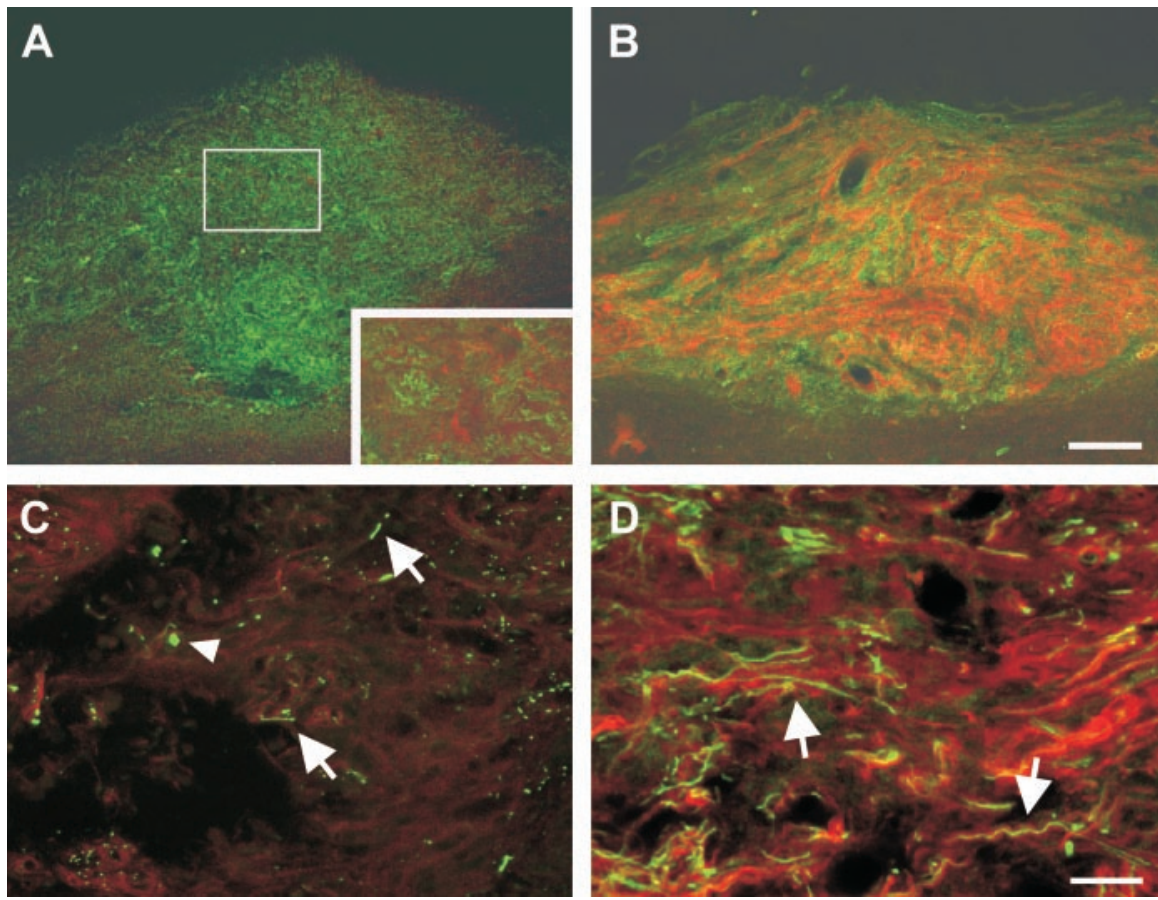

Figure 3. Immunohistochemical characterization of the lesion area at 4 months after injury. $A$, $B$, Staining for fibronectin (green) was used as a marker to visualize scar-associated meningeal cells and fibroblasts in the lesion area and combined with immunohistochemistry for $\mathrm{p} 75^{\mathrm{NTR}}$ (red) as a general antigenic marker for implanted $0 \mathrm{EG}$. $A$, In unimplanted controls, fibronectin was highly expressed in the lesion area with only little immunoreactivity for $p 75^{\text {NTR }}$. Fibronectin-positive cells were found to fill the lesion gap, and virtually no cystic cavity formation was observed. Most of the p75 ${ }^{\text {NTR }}$-stained cells displayed typical bipolar Schwann cell morphology (inset is a higher magnification of the outlined area) that may have migrated into the lesion area from the dorsal root. $B$, Abundant and elevated $\mathrm{p} 75^{\mathrm{NTR}}$ immunoreactivity was found in the lesion area of all OEG implanted animals, indicating that apart from the endogenous Schwann cells present, grafted cells did survive in the lesioned spinal cord. $C, D$, Representative confocal microscope images of the lesion center of an unimplanted control and typical OEG-implanted rat, respectively, at higher magnification. Immunohistochemistry for $75^{\mathrm{NTR}}$ (red) was combined with staining for neurofilament (green) to visualize all axons present in this area. C, Few axons were detected in the lesion center of unimplanted controls (arrows), some of them displaying typical end bulb-like structures (arrowhead). D, In all OEG-implanted rats, numerous NF-positive axons were found in the graft area, often aligning with bipolar p $75^{\text {NTR }}$-positive cells (arrows). This indicates that several unidentified populations of spinal axons were able to regenerate through the OEG implantation site. Scale bars: $A, B, 100 \mu \mathrm{m} ; C, D, 25 \mu \mathrm{m}$.

presumably implanted OEG, together with some endogenous Schwann cells were surrounded by small fibronectin-positive cells, most likely derived from the meningeal sheets (Pasterkamp et al., 1999). When combined with axonal staining, few NFpositive axon-like structures were found in the lesion area of unimplanted controls (Fig. 3C), whereas a dense network of NFpositive axons was detected in OEG-implanted rats, often in close association with p75 ${ }^{\mathrm{NTR}}$-positive cells (Fig. $3 D$ ).

Anterograde tracing revealed that implantation of OEG did not result in robust long-distance regeneration of RST fibers under any condition. In unimplanted animals, the vast majority of traced RST axons (black) displayed "end bulb"-like structures at the proximal border of the lesion site, in the GFAP-positive (brown) area, and did not penetrate into the central component of the scar tissue (Fig. $4 A, C$ ). In animals that received an implant of uninfected, AdV-LacZ- or AdV-NT-3-transduced OEG, respectively, no obvious difference in the distribution of RST axons was observed compared with nonimplanted animals (Fig. $4 B, D, E)$. However, extensive BDNF-mediated sprouting of RST axons was observed in animals that received an OEG implant that was transduced with AdV-BDNF alone or together with AdVNT-3. Sprouting of RST axons preferentially occurred along the interface of the injury site and astrocytic scar (Fig. $4 F$ ). In many cases, aberrant and undirectional sprouting of RST axons was ob- served (Fig. 4G,I). Sprouting of RST axons was primarily restricted to the lesion area, with a small number of fibers extending beyond the lesion and implantation site in the distal spinal cord. However, most of these axons were still in close proximity of the injury site (Fig. $4 H, J)$. From each experimental group, camera lucida drawings through the lesion area of a representative animal were generated to visualize the spatial relationship of traced RST axons to the lesion area (Fig. 5). Microscopic observations were confirmed by quantitative analysis of the regenerative response of rubrospinal neurons to different OEG implants (Fig. 6). Fiber counts of BDA-traced RST axons revealed that no statistical differences were present between animal groups that received either no treatment $(n=6)$ with an implant of uninfected OEG $(p=0.52 ; n=9)$ or AdVLacZ-transduced OEG ( $p=0.12 ; n=6)$, respectively. AdV vector-mediated hypersecretion of NT-3 from transduced OEG also failed to induce growth of RST axons or counteract axonal "dieback" ( $p \geq 0.28$; $n=$ $7)$. In all cases, a similar distribution pattern and dieback of RST axons was observed; although, a consistent tendency toward limited local sprouting seemed present in these OEG implanted groups. These data indicate that RST axons were not overly attracted by OEG implants, and an additional AdV vector-mediated expression of NT-3 by implanted cells did not evoke a regenerative response of RST axons. As expected from the histological observations, a statistically significant BDNF-mediated sprouting of RST axons was found in animal groups $4(p \leq$ $0.0002 ; n=5)$ and $6(p \leq 0.004 ; n=7)$ compared with all other animal groups. No statistical differences were found between these two groups ( $p=0.33$ ), indicating that coexpression of BDNF and NT-3 had no synergistic or counteracting effect on the level of RST axon sprouting.

Volumetric analysis of the injury site revealed that a consistent trend toward smaller lesion volumes was present in all OEG implanted rats compared with controls (Fig. 7). However, no statistically significant differences from unimplanted controls $(n=6)$ were reached in animals implanted with uninfected OEG $(p=$ $0.08 ; n=9)$ or AdV-LacZ-transduced OEG $(p=0.11 ; n=6)$, respectively. In contrast, lesion volumes were almost reduced by half in animals that received OEG implants transduced with AdV vectors encoding BDNF $(p=0.008 ; n=5), \mathrm{NT}-3(p=0.01 ; n=$ $9)$, or a mixture of both vectors $(p=0.004 ; n=7)$. This indicates that AdV vector-mediated secretion of BDNF and NT-3 counteracted secondary degeneration of neural tissue after experimental spinal cord injury.

\section{MEP analysis}

In unoperated age-matched controls, unilateral stimulation of the red nucleus resulted in the recording of a few detectable peaks at the level of cervical vertebra C6 (Fig. 8). The first peak represents the direct projection from the red nucleus, and its amplitude (a direct measure for the number of RST axons) is signifi- 
cantly decreased after transection of the dorsolateral funiculus $(p<0.001)$. The other detectable peaks represent different unidentified descending spinal pathways that are either costimulated during MEP recording or part of the larger motor system that involves the red nucleus. In line with our histological observations, no significant differences in rubrospinal MEP recordings were found among different experimental animal groups $(p>0.05$; data not shown), indicating the absence of robust long-distance regeneration of axons beyond OEG implants (Fig. 8).

\section{Analysis of functional recovery}

Behavioral analysis of spinal cord-injured rats during overground locomotion (BBB score) did not reveal detectable functional deficits in the open field. Although a transient impairment was observed during the initial postoperative phase in both the left forelimb and hindlimb, animals seem to fully recover from the injury regardless of treatment (data not shown). This demonstrates that the $\mathrm{BBB}$ rating score is an inappropriate assay for evaluation of hindlimb function recovery after partial transection of the dorsolateral funiculus, as stated previously (Kim et al., 2001). In contrast, a persistent impairment in especially ipsilateral hindlimb function was detected during horizontal rope walking, emanating in slips of the hindlimb or even a fall from the rope (Fig. 9A). Such impairment was not present during pretraining of the animals before spinal injury. In all operated animals, remaining deficits in general locomotion and hindlimb performance were observed. However, a statistically significant main treatment effect in deficit scores was found ( $p \leq 0.0002$ ) (Fig. 9B). This indicates that all OEG implanted rats had a consistently improved locomotion and slipped or fell from the rope less frequently than unimplanted control animals. As expected, no differences in locomotor behavior were found between animals that received an implant of uninfected or AdVLacZ-transduced OEG, respectively ( $p=$ 0.99). This demonstrates that ex vivo transduction of OEG itself, using AdV vector-mediated gene transfer, did not interfere with the behavioral outcome. In addition, rope locomotion behavior was consistently better in animals that received an OEG implant that was transduced with a neurotrophin-encoding $\mathrm{AdV}$ vector $(p \leq 0.00002)$. Apart from the fact that these rats made fewer errors during rope traverse, other locomotion aspects, in particular body posture and hindpaw placement, were superior in these groups com-
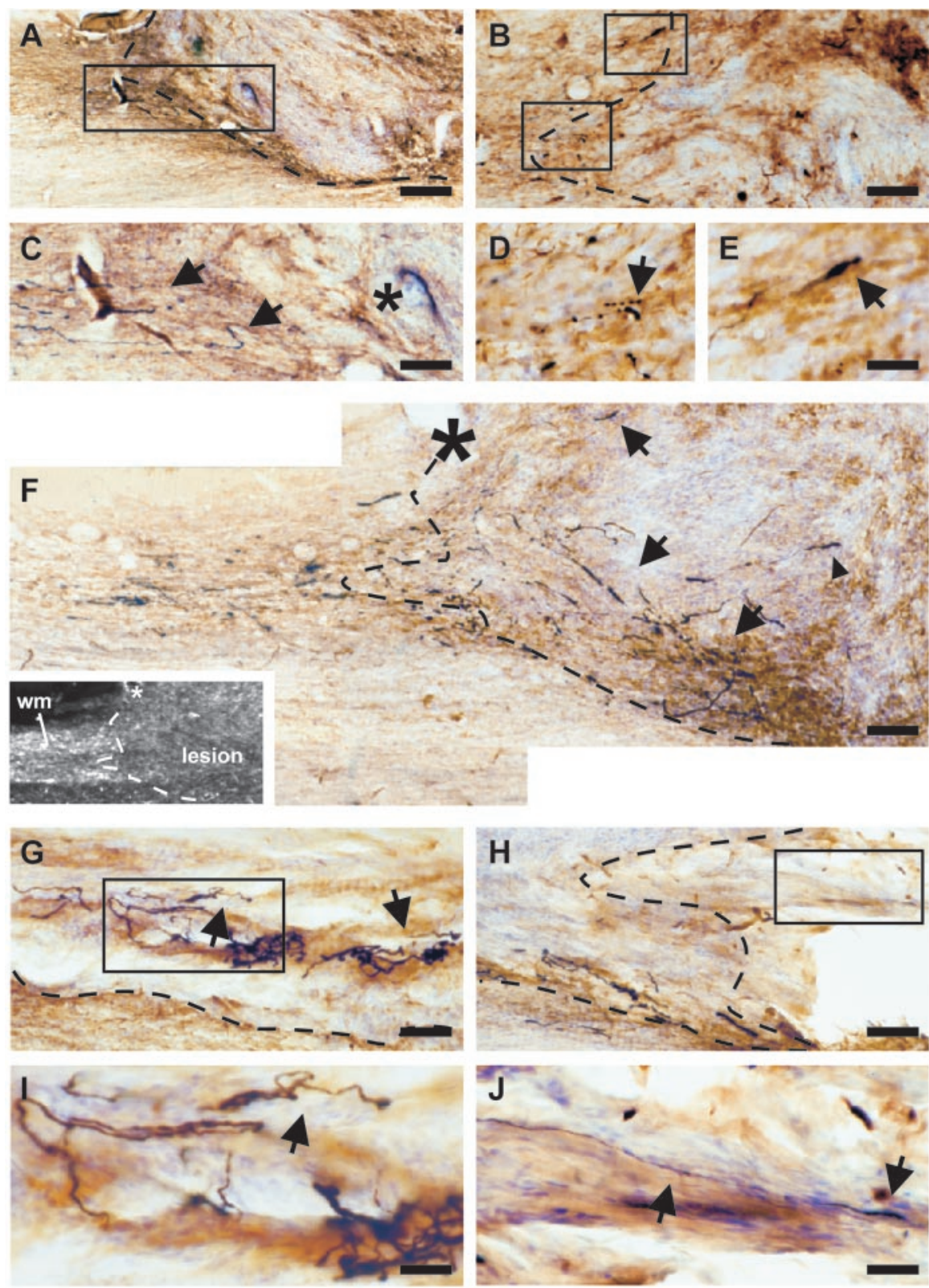

Figure 4. The regenerative response of lesioned RST axons to different types of AdV vector-transduced OEG implants 4 months after injury. All experimental animals were traced with BDA, which was iontophoretically applied 2 weeks before perfusion. Representative photomicrographs of horizontal sections through the cervical spinal cord show anterograde BDA-traced RST axons (black) and reactive GFAP-positive astrocytes (brown) at the lesion and OEG implantation site. DAB double staining was combined with cresyl violet counterstaining (blue) to better visualize tissue cytoarchitecture. In all cases, left is rostral (proximal to the injury), and dashed lines indicate graft-host interfaces. $A, C$, Traced RST axons proximal to the lesion in an unimplanted control animal. C, Higher magnification of the outlined area in $A$, showing that RST axons (arrows) did not regenerate into the lesion area (asterisk), and all stopped at the proximal border of the scar delineated by GFAP-positive astrocytes. B, Termination of lesioned BDA-traced RST axons at the lesion site after implantation of AdV-LacZ-transduced OEG. No sprouting of the RST into the graft and lesion area was observed. $D, E$, Higher magnifications of the corresponding areas outlined in $B$, showing end bulb-like axon endings at the proximal graft-host boundary (arrows). Similar distribution patterns of traced RST axons were found when OEG had been transduced with no or AdV-NT-3 vector before implantation. F-J, RST axon sprouting in representative animals after implantation of AdV-BDNF-transduced OEG. F, RST axon regeneration into the graft was observed. The dashed line indicates the rostral graft-host interface as determined using dark-field microscopy (inset; asterisk, blood vessel; wm, white matter). Sprouting preferentially seemed to occur along the graft-host interface (arrows) with some axons present in the central core of the lesion and implantation site (arrowhead). $G$, Traced RST axons deeper in the graft (arrows). H, BDA-labeled RST axons at the caudal graft-host interface. I, Higher magnification of the outlined area in G, showing undirectional growth of RST axons (arrow). J, Higher magnification of corresponding area in $H$. Few axons were found to leave the graft and extend distally into the host spinal cord (arrow). Scale bars: A, $220 \mu \mathrm{m} ; B, C, F-H, 92 \mu \mathrm{m} ; D, E, 36 \mu \mathrm{m} ; I, J, 23 \mu \mathrm{m}$. 

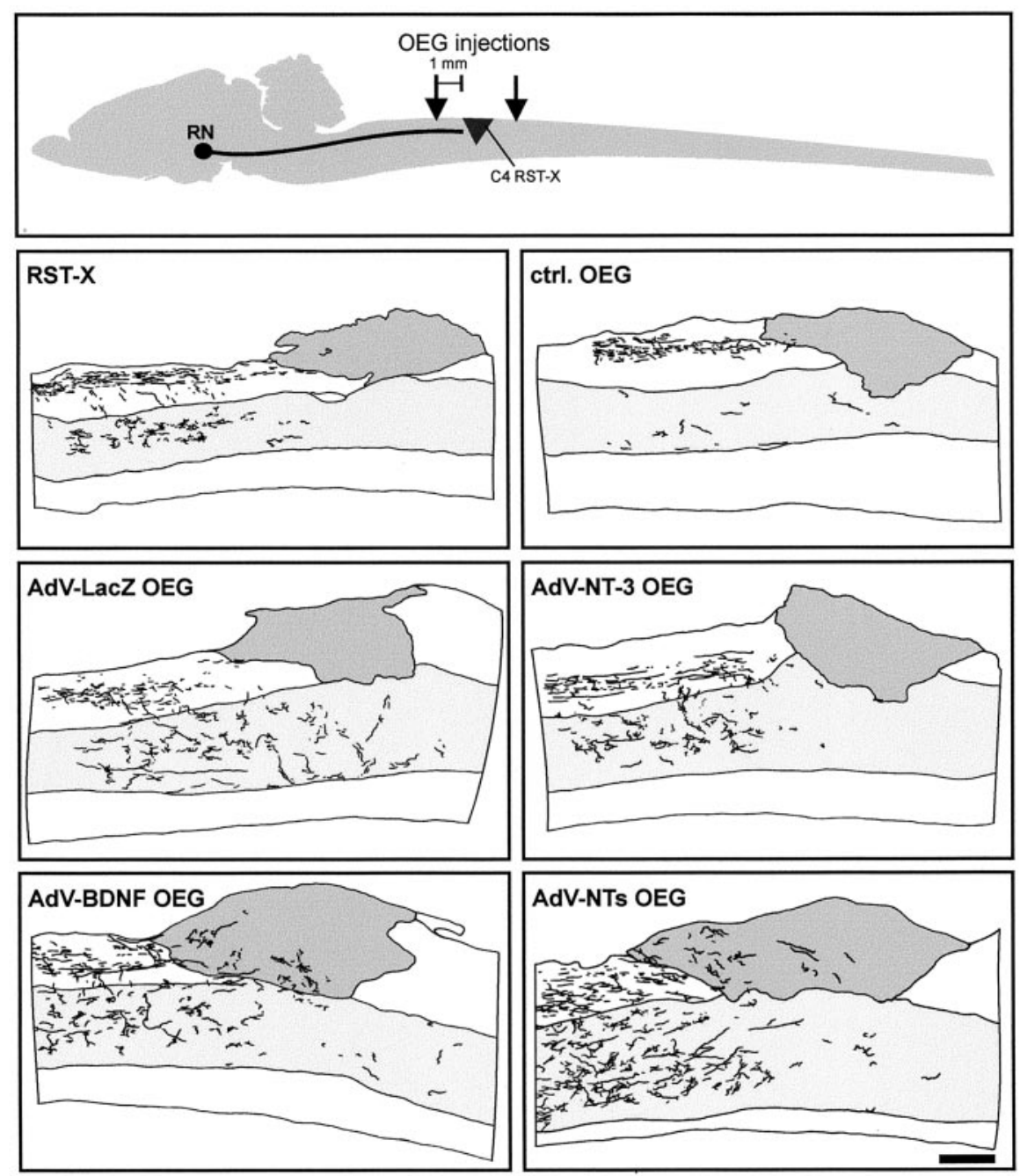

Figure 5. Graphic representation of the regenerative response of BDA-traced RST axons after implantation of AdV vectortransduced OEG 4 months after injury. Top, Schematic representation of spinal cord lesion model and OEG injection sites. RN, Red nucleus. Camera lucida drawings made of representative animals from each experimental group are shown. Note the distribution pattern of BDA-traced RST axons (black) and their collaterals in the spinal cord gray matter (light gray area) at the injury site (gray area). The latter contained both scar-associated cells and implanted OEG. NTs, BDNF plus NT-3. Scale bar, $450 \mu \mathrm{m}$.

pared with animals from control groups (lesion only or control OEG implant) (Fig. 9A). These observations were reflected in the significantly lower deficit scores. Interestingly, within all experimental groups, no significant additional improvements in functional performance were found after the first month after surgery. This indicates that recovery of function primarily occurred during the first 4 weeks after injury and OEG implantation.

Calculated total error/step ratios were consistent with the behavioral data as obtained from the deficit-scoring method (Fig. 9C). Significant treatment-based differences were found among the experimental groups. All OEG-implanted animal groups displayed an improved recovery after the injury resulting in significantly lower error scores (fewer slips or falls) than unimplanted lesioned control rats $(p \leq 0.0003)$. Again, no statistical difference $(p=0.57)$ in horizontal rope locomotion performance was found among rats that received an implant of either uninfected or AdVLacZ-transduced OEG. Enhanced recovery was observed in animal groups that received OEG implants subjected to transduction with neurotrophin-encoding AdV vector. When compared with AdVLacZ-transduced OEG implanted rats, fewer errors were observed in animals implanted with AdV-BDNF-transduced OEG, but this just did not reach statistical significance $(p=$ 0.07). However, statistically significant improvements were obtained after implantation of AdV-NT-3 OEG ( $p=0.02)$ or AdVBDNF/NT-3-transduced OEG $(p=0.009)$. Together, these data demonstrate that neurotrophin-encoding AdV vectortransduced OEG implants showed the best recovery after injury, whereas all OEGimplanted animal groups were significantly less impaired than unimplanted lesioned controls.

Interestingly, a significant positive correlation existed between the error scores of the impaired (left) hindpaw of individual animals and accompanying lesion volumes (Pearson's correlation test; $r=$ $0.632 ; p<0.001)$. Rats that performed well during horizontal rope walking (low error score) were found to have relatively small lesions, whereas animals that slipped or fell from the rope more frequently consistently had bigger lesion volumes (Fig. 9D). This suggests that possible neuroprotective effects of BDNF and NT-3, counteracting secondary degeneration, positively influenced the functional performance of animals during horizontal rope locomotion, which would also be in line with the time course of recovery.

\section{Discussion}

The present study uniquely combined OEG implantation with ex vivo AdV vector-mediated neurotrophin gene transfer. We demonstrate the following: (1) OEG implants transduced with neurotrophinencoding AdV vectors augmented enhanced recovery of hindlimb function compared with controls as determined by behavioral testing. (2) Lesion volumes were smaller in OEG-implanted animals and significantly reduced in size in rats receiving a graft that was ex vivo transduced with neurotrophin-encoding AdV vector. (3) A positive correlation did exist between functional impairment and lesion size, indicating that reduction of secondary damage by OEG implants leads to an improved functional outcome. (4) Extensive BDNF-stimulated sprouting of RST axons was induced up to $1-1.5 \mathrm{~mm}$ into the lesion area, but there was no obvious correlation with behavioral data. Quantitative histological and electrophysiological analysis showed that OEG did not induce major long-distance RST regeneration. Therefore, the correlation between lesion size and functional performance indicates that the recovery was at least partially related to protective effects of OEG implants on the surrounding spinal cord.

\section{Functional performance correlates with lesion size}

All OEG-implanted animals displayed better functional performance during horizontal rope walking, which was also enhanced in rats receiving an implant of neurotrophin-encoding AdV vector-transduced OEG. Functional studies on the physiological role of the red nucleus have demonstrated that its specific ablation results in persistent, although minor, deficits in hindlimb 
functioning (Muir and Whishaw, 2000). This suggests that the observed impairments in hindlimb performance during horizontal rope walking were not only attributable to a loss of rubral input. Damage to other motor pathways in the lateral funiculus (Holstege, 1991) likely contributed to these remaining deficits. The striking correlation between lesion size and functional performance indicates that the degree of recovery was likely related to the protective potential of OEG implants, which is a novel finding. Recently, OEGmediated tissue sparing has been described in a contusion injury model (Takami et al., 2002; Plant et al., 2003). However, it remained to be elucidated whether this also improved functional recovery. A significant reduction in lesion size was found only after implantation of OEG transduced with neurotrophin-encoding AdV vectors. This indicates that BDNF and NT-3 counteracted secondary tissue degeneration. Such a restorative role for neurotrophins was proposed previously. BDNF has been shown to reduce tissue necrosis after spinal cord injury (Novikova et al., 1996). This feature is important because spinal tissue sparing, in particular ventrolateral white matter, has been directly related to the locomotor capacity of spinal cord-injured rats (Schucht et al., 2002). Furthermore, both BDNF and NT-3 have been found to induce oligodendrocyte proliferation and remyelination of damaged axons (McTigue et al., 1998), which is of interest because remaining deficits after injury are partly caused by demyelination of spared axons.

Together, we demonstrate that lesion size directly appeared to influence impaired hindlimb performance. This supports the idea that neurotrophin-mediated tissue preservation after implantation of AdV vector-transduced OEG in the subacute phase after injury contributed to the level of functional recovery rather than regeneration of the RST itself.

\section{AdV vector-transduced OEG implants and RST regeneration}

Genetic modification allowed us to manipulate the growthpromoting properties of OEG. In vitro, transduction of OEG with AdV-BDNF or AdV-NT-3 resulted in a cell type that was more effective in promoting neurite growth. In vivo, only AdV-BDNFtransduced OEG were capable of inducing a significant regenerative response of RST axons. In previous work, using ex vivo lentiviral vector-transduced OEG expressing the marker protein green fluorescent protein to reliably track implanted cells, we unequivocally demonstrated that implanted OEG did survive in the lesioned spinal cord at least up to 4 months (Ruitenberg et al., 2002). This indicates that under these conditions and in the absence of elevated BDNF levels, lesioned RST axons do not robustly respond to OEG implants. To date, a number of axon populations were reported to regenerate through OEG implants in different injury models, including sensory axons of the dorsal root axons (Ramon-Cueto and Nieto-Sampedro, 1994; Navarro et al., 1999) as well as serotonergic axons (Ramon-Cueto et al., 1998, 2000; Lu et al., 2001a, 2002) and CST axons (Li et al., 1998; Ramon-Cueto et al., 2000). Some controversy has arisen on the regenerative response of the CST because others found that OEGinduced sprouting was limited to the proximal injury site as observed here for the RST (Takami et al., 2002; D. B. Levison, M. J. Ruitenberg, G. W. Plant, unpublished observations). It is therefore important to obtain more insight in both OEG cell biology and specific neurotrophic requirements of the different spinal pathways. Extended knowledge of proteins involved in regeneration of specific nerve tracts will allow the development of more optimal bridging substrates via genetic engineering.

Intraparenchymal infusions of BDNF induce enhanced regeneration of supraspinal axons, including the RST (Xu et al., 1995; Kobayashi et al., 1997). However, a gene therapy-based strategy to deliver neurotrophins has several advantages over the use of osmotic minipumps (for review, see Dijkhuizen and Verhaagen, 1999). To date, engineered Schwann cells (Menei et al., 1998) and fibroblasts (Liu et al., 1999; Lu et al., 2001b) have been used to deliver BDNF to the injured spinal cord. Vigorous growth of RST axons was reported after implantation of BDNF-secreting fibroblasts (Liu et al., 1999). In the present study, only short-range BDNF-mediated sprouting was observed. This discrepancy in experimental outcome between our study and the findings of Liu et al. (1999) is likely explained by several differences in treatment conditions (i.e., the duration of transgene expression and num- 

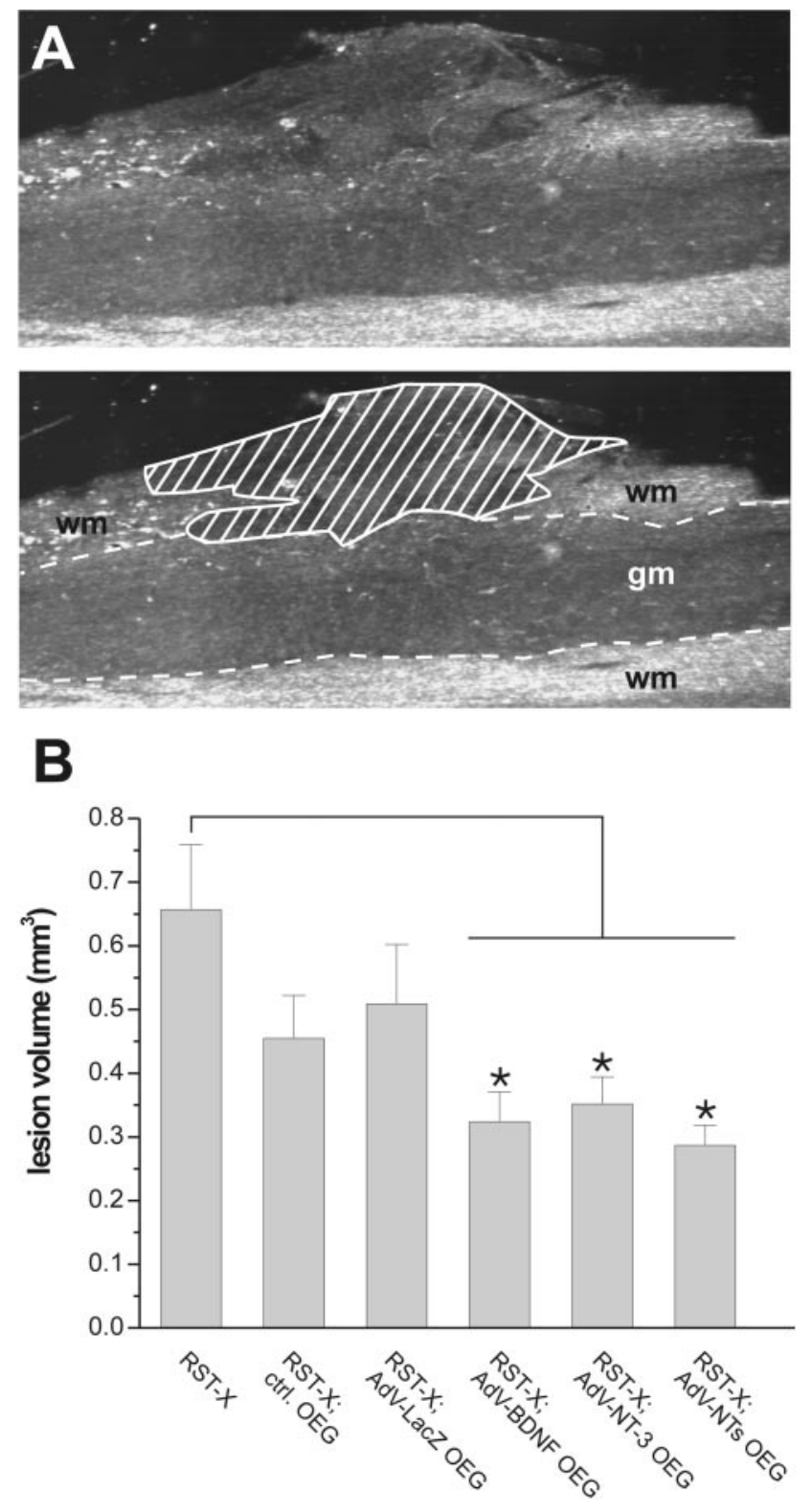

Figure 7. OEG implants reduce lesion size after implantation in the lesioned rat spinal cord. $A$, Example of lesion size determination in an OEG implanted animal using dark-field microscopy. Top, A discontinuity of normal spinal cord cytoarchitecture and a high density of small cells that invaded the injury site clearly defined the borders of the damaged area. Bottom, Computerized image analysis picture showing the outlined damaged (shaded) area to determine lesion size. gm, Gray matter; wm, white matter. B, Graphic representation showing lesion volumes in cubic millimeters (average $\pm S E M$ ). A consistent trend toward smaller lesion volumes was found in all OEG-implanted animals. Significant reduction in lesion size was found only after implantation of AdV-BDNF-, AdV-NT-3-, or AdV-BDNF plus AdV-NT-3-transduced OEG, respectively. Asterisks indicate statistically significant differences $(p<0.05)$ from lesioned unimplanted controls.

ber of implanted cells). Most importantly, AdV vector-mediated transduction of OEG results in a transient time course of transgene expression after implantation in the lesioned spinal cord (up to $30 \mathrm{~d}$ ) (Ruitenberg et al., 2002), whereas persistent expression and thereby continuous neurotrophic support is provided by engineered fibroblast grafts. In future studies, it is therefore important to study RST regeneration after implantation of stable transduced OEG [e.g., using lentiviral vector-mediated gene transfer, which results in persistent transgene expression (Ruitenberg et al., 2002), and compare those implants directly to grafts of engi-

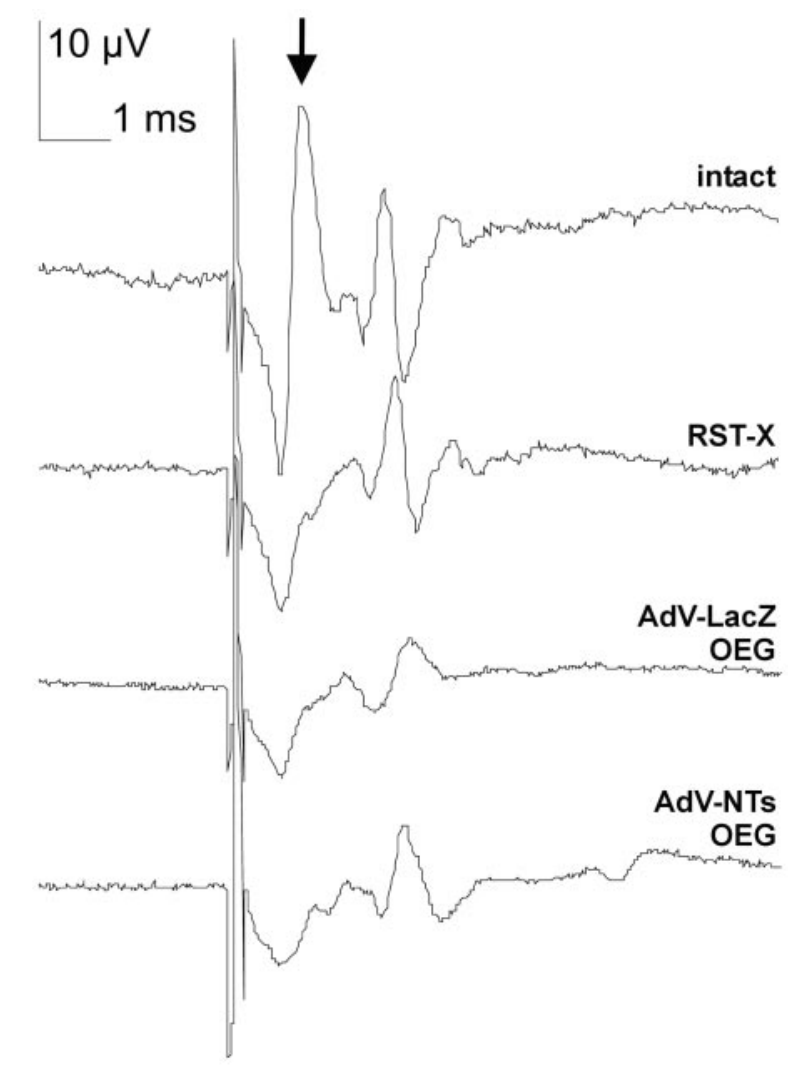

Figure 8. Four examples of representative rubrospinal MEP recordings from an intact and lesioned control and after AdV vector-transduced $0 \mathrm{EG}$ implantation. After unilateral transection of the dorsolateral funiculus, a significant decrease $(p<0.001)$ in the amplitude of the first peak was observed in all groups compared with age-matched unlesioned control animals (arrow). No treatment-based statistical differences in amplitude of this peak were observed among experimental groups, which indicates the absence of robust and functional RST regeneration. Calibration: $10 \mu \mathrm{V}, 1 \mathrm{msec}$.

neered fibroblasts]. Ideally, neurotrophin expression should be regulated to allow modulation of neurite outgrowth (Blesch et al., $2000,2001)$ and prevent entrapment of axons in areas with high neurotrophic factor expression (Kirik et al., 2000).

Sprouting of RST axons preferentially occurred along the interface of the lesion area and intact spinal cord. This was also observed by Liu et al. (1999) and suggests that, in the presence of elevated neurotrophin levels, reactive glia may act as an attractive area for lesioned axons to grow (Kawaja and Gage, 1991). Alternatively, RST axons may try to avoid the lesion core that, besides implanted OEG, also contains scar-associated cells (e.g., meningeal cells). Several growth inhibitory molecules have been associated with these cells and may repel growing RST axons from this area (for review, see Fawcett and Asher, 1999; Pasterkamp et al., 1999; De Winter et al., 2002).

Implantation of NT-3-producing OEG did not elicit a growth response of axotomized RST axons. Although the receptors for BDNF and NT-3, TrkB and TrkC, are coexpressed on rubrospinal neurons, these neurotrophins seem to have distinct roles after injury. NT-3 reportedly counteracts injury-induced death in newborn rats (Diener and Bregman, 1994) but, in contrast to BDNF, does not elicit regeneration-associated gene expression or reverse lesion-induced atrophy during adulthood (Kobayashi et al., 1997). This suggests a role for NT-3 in survival of RST neurons rather than axonal regeneration, which is opposite for the CST, in which BDNF was shown to promote survival but not regeneration (Lu et al., 2001b). 
After implantation of a peripheral nerve graft, regeneration of RST fibers has been observed (Richardson et al., 1984). Despite the fact that Schwann cells appear more potent growth promoters for RST axons, evidence is emerging that they do not interact well with a CNS environment and upregulate inhibitory chondroitinsulfate proteoglycan expression in astrocytes (Plant et al., 2001; Takami et al., 2002). Therefore, OEG could be better candidates as cellular platforms for ex vivo gene transfer to repair the injured spinal cord. Furthermore, in contrast to genetically modified fibroblasts, OEG are of CNS origin and normally express a subset of factors that support neurite outgrowth (for review, see Ramon-Cueto and Avila, 1998; Woodhall et al., 2001).

The combination of neural transplantation and neurotrophin delivery has emerged as a promising strategy to augment regeneration and functional recovery after spinal cord injury (for review, see Bunge, 2001; Blits et al., 2002). We demonstrate that ex vivo transduction of OEG with BDNF- or NT-3-encoding AdV vectors did: (1) significantly reduce lesion size, (2) enhance the growth-promoting properties of these cells, and (3) improve functional recovery after implantation. A limitation of AdV vector-transduced OEG implants is that they did not induce robust growth of RST axons distal from the injury site, which is essential to restore damaged neural networks. Stable transduction of OEG with neurotrophin-encoding lentiviral vectors could significantly improve the results obtained, giving hope for the use of these modified cells in both acute and chronic spinal cord injury models.

\section{References}

Akli S, Caillaud C, Vigne E, Stratford-Perricaudet LD, Poenaru L, Perricaudet M, Kahn A, Peschanski MR (1993) Transfer of a foreign gene into the brain using adenovirus vectors. Nat Genet 3:224-228.

Antal M, Sholomenko GN, Moschovakis AK, Storm-Mathisen J, Heizmann CW, Hunziker W (1992) The termination pattern and postsynaptic targets of rubrospinal fibers in the rat spinal cord: a light and electron microscopic study. J Comp Neurol 325:22-37.

Barnett SC, Alexander CL, Iwashita Y, Gilson JM, Crowther J, Clark L, Dunn LT, Papanastassiou V, Kennedy PG, Franklin RJ (2000) Identification of a human olfactory ensheathing cell that can effect transplant-mediated remyelination of demyelinated CNS axons. Brain 123:1581-1588.

Basso DM, Beattie MS, Bresnahan JC (1995) A sensitive and reliable locomotor rating scale for open field testing in rats. J Neurotrauma 12:1-21.

Blesch A, Uy HS, Diergardt N, Tuszynski MH (2000) Neurite outgrowth can be modulated in vitro using a tetracycline-repressible gene therapy vector expressing human nerve growth factor. J Neurosci Res 59:402-409.

Blesch A, Conner JM, Tuszynski MH (2001) Modulation of neuronal survival and axonal growth in vivo by tetracycline-regulated neurotrophin expression. Gene Ther 8:954-960.

Blits B, Dijkhuizen PA, Boer GJ, Verhaagen J (2000) Intercostal nerve im-
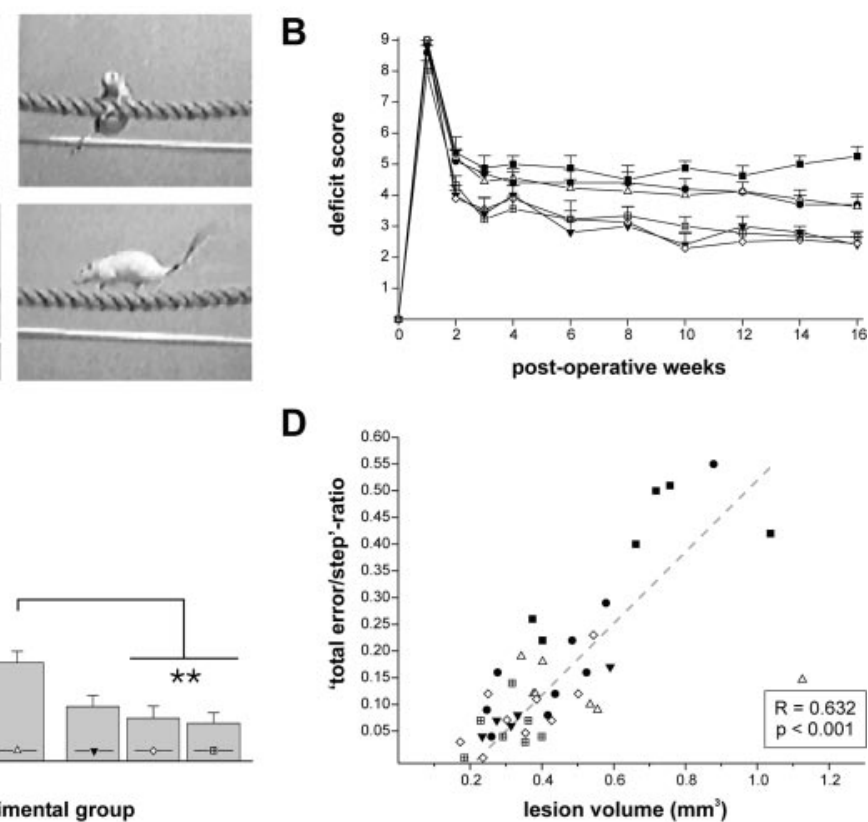

\begin{tabular}{lll}
\hline-- RSTX & $\rightarrow-$ AdV-LacZ OEG & $\rightarrow$ AdV-NT-3 OEG \\
$\rightarrow-$ ctrl. OEG & $\rightarrow-$ AdV-BDNF OEG & $\rightarrow$ AdV-NTs OEG \\
\hline
\end{tabular}

Figure 9. OEG implants promote recovery of hindlimb function during horizontal rope walking. A, Photographs captured from video recordings showing experimental animals crossing the rope 4 months after injury. Top, Typical example of a slip and fall from the rope frequently observed in unimplanted lesioned controls. Bottom, All OEG-implanted rats had better functional perfor列 resulted in a significantly enhanced main effect of the treatment $(p<0.00002)$ compared with all other groups, whereas no

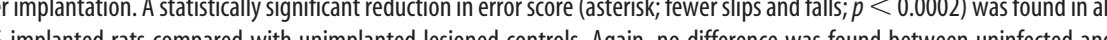
AdV-LacZ-transduced OEG implants ( $p=0.57$ ). Implantation of neurotrophin-encoding AdV vector-transduced OEG resulted in (1) 0.02) or AdV-BDNF plus NT-3 ( $p=0.009$; double asterisks)-transduced OEG. No statistical differences were

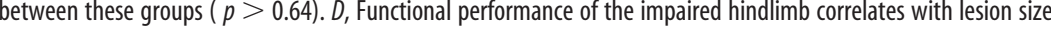
catter graph of calculated total error/step ratios plotted against accompanying lesion volumes shows the existence of a positive correlation between hindlimb function and lesion size as determined by Pearson's correlation test.

plants transduced with an adenoviral vector encoding neurotrophin-3 promote regrowth of injured rat corticospinal tract fibers and improve hindlimb function. Exp Neurol 164:25-37.

Blits B, Boer GJ, Verhaagen J (2002) Pharmacological, cell, and gene therapy strategies to promote spinal cord regeneration. Cell Transplant 11:593-613.

Boruch AV, Conners JJ, Pipitone M, Deadwyler G, Storer PD, Devries GH, Jones KJ (2001) Neurotrophic and migratory properties of an olfactory ensheathing cell line. Glia 33:225-229.

Buijs RM, Wortel J, Van Heerikhuize JJ, Feenstra MG, Ter Horst GJ, Romijn HJ, Kalsbeek A (1999) Anatomical and functional demonstration of a multisynaptic suprachiasmatic nucleus adrenal (cortex) pathway. Eur J Neurosci 11:1535-1544.

Bunge MB (2001) Bridging areas of injury in the spinal cord. Neuroscientist 7:325-339.

De Winter F, Oudega M, Lankhorst AJ, Hamers FP, Blits B, Ruitenberg MJ, Pasterkamp RJ, Gispen WH, Verhaagen J (2002) Injury-induced class 3 semaphorin expression in the rat spinal cord. Exp Neurol 175:61-75. 
Diener PS, Bregman BS (1994) Neurotrophic factors prevent the death of CNS neurons after spinal cord lesions in newborn rats. NeuroReport 5:1913-1917.

Dijkhuizen PA, Hermens WT, Teunis MA, Verhaagen J (1997) Adenoviral vector-directed expression of neurotrophin-3 in rat dorsal root ganglion explants results in a robust neurite outgrowth response. J Neurobiol 33:172-184.

Dijkhuizen PA, Verhaagen J (1999) The use of neurotrophic factors to treat spinal cord injury: advantages and disadvantages of different delivery methods. Neurosci Res Commun 24:1-10.

Doucette R (1990) Glial influences on axonal growth in the primary olfactory system. Glia 3:433-449.

Doucette R (1991) PNS-CNS transitional zone of the first cranial nerve. J Comp Neurol 312:451-466.

Fallaux FJ, Kranenburg O, Cramer SJ, Houweling A, Van Ormondt H, Hoeben RC, Van der Eb AJ (1996) Characterization of 911: a new helper cell line for the titration and propagation of early region 1-deleted adenoviral vectors. Hum Gene Ther 7:215-222.

Farbman A (1992) Cell biology of olfaction. Cambridge, UK: Cambridge UP.

Fawcett JW, Asher RA (1999) The glial scar and central nervous system repair. Brain Res Bull 49:377-391.

Fournier AE, Strittmatter SM (2001) Repulsive factors and axon regeneration in the CNS. Curr Opin Neurobiol 11:89-94.

Franklin RJ, Barnett SC (2000) Olfactory ensheathing cells and CNS regeneration: the sweet smell of success? Neuron 28:15-18.

Giger RJ, Wolfer DP, De Wit GM, Verhaagen J (1996) Anatomy of rat semaphorin III/collapsin-1 mRNA expression and relationship to developing nerve tracts during neuroembryogenesis. J Comp Neurol 375:378-392.

Griffioen HA, Van der Beek E, Boer GJ (1992) Gelatin embedding to preserve lesion-damaged hypothalami and intracerebroventricular grafts for vibratome slicing and immunocytochemistry. J Neurosci Methods 43:43-47.

Grill R, Murai K, Blesch A, Gage FH, Tuszynski MH (1997) Cellular delivery of neurotrophin-3 promotes corticospinal axonal growth and partial functional recovery after spinal cord injury. J Neurosci 17:5560-5572.

Gudino-Cabrera G, Pastor AM, de la Cruz RR, Delgado-Garcia JM, NietoSampedro M (2000) Limits to the capacity of transplants of olfactory glia to promote axonal regrowth in the CNS. NeuroReport 11:467-471.

Hermens WT, Giger RJ, Holtmaat AJ, Dijkhuizen PA, Houweling DA, Verhaagen J (1997) Transient gene transfer to neurons and glia: analysis of adenoviral vector performance in the CNS and PNS. J Neurosci Methods 71:85-98.

Holstege G (1991) Descending motor pathways and the spinal motor system: limbic and non-limbic components. Prog Brain Res 87:307-421.

Houle JD, Ye JH (1999) Survival of chronically-injured neurons can be prolonged by treatment with neurotrophic factors. Neuroscience 94:929-936.

Jones LL, Oudega M, Bunge MB, Tuszynski MH (2001) Neurotrophic factors, cellular bridges and gene therapy for spinal cord injury. J Physiol (Lond) 533:83-89.

Kawaja MD, Gage FH (1991) Reactive astrocytes are substrates for the growth of adult CNS axons in the presence of elevated levels of nerve growth factor. Neuron 7:1019-1030.

Kim D, Schallert T, Liu Y, Browarak T, Nayeri N, Tessler A, Fischer, Murray M (2001) Transplantation of genetically modified fibroblasts expressing BDNF in adult rats with a subtotal hemisection improves specific motor and sensory functions. Neurorehabil Neural Repair 15:141-150.

Kirik D, Rosenblad C, Bjorklund A (2000) Preservation of a functional nigrostriatal dopamine pathway by GDNF in the intrastriatal 6-OHDA lesion model depends on the site of administration of the trophic factor. Eur J Neurosci 12:3871-3882.

Kobayashi NR, Fan DP, Giehl KM, Bedard AM, Wiegand SJ, Tetzlaff W (1997) BDNF and NT-4/5 prevent atrophy of rat rubrospinal neurons after cervical axotomy, stimulate GAP-43 and Talphal-tubulin mRNA expression, and promote axonal regeneration. J Neurosci 17:9583-9595.

Kozak M (1987) At least six nucleotides preceding the AUG initiator codon enhance translation in mammalian cells. J Mol Biol 196:947-950.

Kwon BK, Liu J, Messerer C, Kobayashi NR, McGraw J, Oschipok L, Tetzlaff W (2002) Survival and regeneration of rubrospinal neurons 1 year after spinal cord injury. Proc Natl Acad Sci USA 99:3246-3251.
Lankhorst AJ, Duis SE, ter Laak MP, Joosten EA, Hamers FP, Gispen WH (1999) Functional recovery after central infusion of alpha-melanocytestimulating hormone in rats with spinal cord contusion injury. J Neurotrauma 16:323-331.

Li Y, Field PM, Raisman G (1997) Repair of adult rat corticospinal tract by transplants of olfactory ensheathing cells. Science 277:2000-2002.

Li Y, Field PM, Raisman G (1998) Regeneration of adult rat corticospinal axons induced by transplanted olfactory ensheathing cells. J Neurosci 18:10514-10524.

Liebl DJ, Huang W, Young W, Parada LF (2001) Regulation of Trk receptors following contusion of the rat spinal cord. Exp Neurol 167:15-26.

Liu Y, Kim D, Himes BT, Chow SY, Schallert T, Murray M, Tessler A, Fischer I (1999) Transplants of fibroblasts genetically modified to express BDNF promote regeneration of adult rat rubrospinal axons and recovery of forelimb function. J Neurosci 19:4370-4387.

Lu J, Feron F, Mackay-Sim A, Waite PM (2002) Olfactory ensheathing cells promote locomotor recovery after delayed transplantation into transected spinal cord. Brain 125:14-21.

Lu J, Feron F, Ho SM, Mackay-Sim A, Waite PM (2001a) Transplantation of nasal olfactory tissue promotes partial recovery in paraplegic adult rats. Brain Res 889:344-357.

Lu P, Blesch A, Tuszynski MH (2001b) Neurotrophism without neurotropism: BDNF promotes survival but not growth of lesioned corticospinal neurons. J Comp Neurol 436:456-470.

McTigue DM, Horner PJ, Stokes BT, Gage FH (1998) Neurotrophin-3 and brain-derived neurotrophic factor induce oligodendrocyte proliferation and myelination of regenerating axons in the contused adult rat spinal cord. J Neurosci 18:5354-5365.

Menei P, Montero-Menei C, Whittemore SR, Bunge RP, Bunge MB (1998) Schwann cells genetically modified to secrete human BDNF promote enhanced axonal regrowth across transected adult rat spinal cord. Eur J Neurosci 10:607-621.

Muir GD, Whishaw IQ (2000) Red nucleus lesions impair overground locomotion in rats: a kinetic analysis. Eur J Neurosci 12:1113-1122.

Murray M, Fischer I (2001) Transplantation and gene therapy: combined approaches for repair of spinal cord injury. Neuroscientist 7:28-41.

Nathan PW (1994) Effects on movement of surgical incisions into the human spinal cord. Brain 117:337-346.

Navarro X, Valero A, Gudino G, Fores J, Rodriguez FJ, Verdu E, Pascual R, Cuadras J, Nieto-Sampedro M (1999) Ensheathing glia transplants promote dorsal root regeneration and spinal reflex restitution after multiple lumbar rhizotomy. Ann Neurol 45:207-215.

Novikova L, Novikov L, Kellerth JO (1996) Brain-derived neurotrophic factor reduces necrotic zone and supports neuronal survival after spinal cord hemisection in adult rats. Neurosci Lett 220:203-206.

Pasterkamp RJ, Giger RJ, Ruitenberg MJ, Holtmaat AJ, De Wit J, De Winter F, Verhaagen J (1999) Expression of the gene encoding the chemorepellent semaphorin III is induced in the fibroblast component of neural scar tissue formed following injuries of adult but not neonatal CNS. Mol Cell Neurosci 13:143-166.

Plant GW, Bates ML, Bunge MB (2001) Inhibitory proteoglycan immunoreactivity is higher at the caudal than the rostral Schwann cell grafttransected spinal cord interface. Mol Cell Neurosci 17:471-487.

Plant GW, Christensen CL, Oudega M, Bunge MB (2003) Delayed transplantation of olfactory ensheathing glia promotes sparing/regeneration of supraspinal axons in the contused adult rat spinal cord. J Neurotrauma 20:1-16.

Plant GW, Ramon-Cueto A, Bunge MB (2000) Transplantation of Schwann cells and ensheathing glia to improve regeneration in adult spinal cord. In: Axonal regeneration in the central nervous system (Ingoglia NA, Murray M, eds), pp 529-561. New York: Marcel Dekker.

Ramon-Cueto A, Nieto-Sampedro M (1994) Regeneration into the spinal cord of transected dorsal root axons is promoted by ensheathing glia transplants. Exp Neurol 127:232-244.

Ramon-Cueto A, Plant GW, Avila J, Bunge MB (1998) Long-distance axonal regeneration in the transected adult rat spinal cord is promoted by olfactory ensheathing glia transplants. J Neurosci 18:3803-3815.

Ramon-Cueto A, Avila J (1998) Olfactory ensheathing glia: properties and function. Brain Res Bull 46:175-187.

Ramon-Cueto A, Cordero MI, Santos-Benito FF, Avila J (2000) Functional 
recovery of paraplegic rats and motor axon regeneration in their spinal cords by olfactory ensheathing glia. Neuron 25:425-435.

Richardson PM, Issa VM, Aguayo AJ (1984) Regeneration of long spinal axons in the rat. J Neurocytol 13:165-182.

Ruitenberg MJ, Plant GW, Christensen CL, Blits B, Niclou SP, Harvey AR, Boer GJ, Verhaagen J (2002) Viral vector-mediated gene expression in olfactory ensheathing glia implants in the lesioned rat spinal cord. Gene Ther 9:135-146.

Schucht P, Raineteau O, Schwab ME, Fouad K (2002) Anatomical correlates of locomotor recovery following dorsal and ventral lesions of the rat spinal cord. Exp Neurol 176:143-153.

Schnell L, Schneider R, Kolbeck R, Barde YR, Schwab ME (1994) Neurotrophin-3 enhances sprouting of corticospinal tract during development and after spinal cord lesion. Nature 367:170-173.

Schwab ME, Bartholdi D (1996) Degeneration and regeneration of axons in the lesioned spinal cord. Physiol Rev 76:319-370.
Takami T, Oudega M, Bates ML, Wood PM, Kleitman N, Bunge MB (2002) Schwann cell but not olfactory ensheathing glia transplants improve hindlimb locomotor performance in the moderately contused adult rat thoracic spinal cord. J Neurosci 22:6670-6681.

Tetzlaff W, Kobayashi NR, Giehl KM, Tsui BJ, Cassar SL, Bedard AM (1994) Response of rubrospinal and corticospinal neurons to injury and neurotrophins. Prog Brain Res 103:271-286.

Woodhall E, West AK, Chuah MI (2001) Cultured olfactory ensheathing cells express nerve growth factor, brain-derived neurotrophic factor, glia cell line-derived neurotrophic factor and their receptors. Brain Res Mol Brain Res 88:203-213.

Xu XM, Guenard V, Kleitman N, Aebischer P, Bunge MB (1995) A combination of BDNF and NT-3 promotes supraspinal axonal regeneration into Schwann cell grafts in adult rat thoracic spinal cord. Exp Neurol 134:261-272. Yan H, Bunge MB, Wood PM, Plant GW (2001) Mitogenic response of adult rat olfactory ensheathing glia to four growth factors. Glia 33:334-342. 